\title{
Two-level layup optimization of composite laminate using lamination parameters
}

\author{
Xiaoyang Liu, Carol A. Featherston and David Kennedy* \\ School of Engineering, Cardiff University, Queen's Buildings, The Parade, Cardiff CF24 3AA, UK \\ * Corresponding author. Email KennedyD@cardiff.ac.uk
}

\begin{abstract}
This paper provides an efficient method for performing global layup optimization of composite laminates with buckling and manufacturing constraints. The optimization problem is divided into two stages and is based on the use of lamination parameters. During the first stage, exact finite strip analysis and continuous optimum design are employed for buckling optimization of the lamination parameters and laminate thickness. In the second stage, a logic-based procedure combining the branch and bound method with a global layerwise technique is employed to find the optimal stacking sequences to match the optimized lamination parameters obtained in the first stage. In order to ensure the optimized layup can be used in practice, four manufacturing constraints are added into the logical search process, and the feasible region for the lamination parameters with a manufacturing constraint which requires at least $10 \%$ of each of four possible ply orientations is studied. By comparing the logic-based method with the use of a genetic algorithm for searching stacking sequences under different requirements, the high efficiency and ability to achieve a global optimal result of the logic-based method are demonstrated.
\end{abstract}

Keywords: composite, layup optimization, lamination parameters, branch and bound, manufacturing constraints

\section{Introduction}

Laminated composite structures are increasingly being adopted in aerospace structures because of their high specific strength and stiffness ratios as well as the fact that they offer the advantage over conventional aircraft materials of being able to be tailored for a particular application [1]. Consideration of design cost and manufacturing limitations means that fibre orientations are usually restricted to $0^{\circ}, 90^{\circ},+45^{\circ}$ and $-45^{\circ}$ in the optimum design of constant stiffness composite laminates.

Normally, there are three successive stages in aircraft design [2]: conceptual design, preliminary design and detailed design. The most powerful analysis methods including Finite Element Analysis (FEA) are employed in the detailed design stage to provide complete design information for configurations chosen in the preliminary stage. However, such techniques are quite time-consuming and require a large computational resource making them unsuitable in preliminary design in which many candidate configurations need to be analysed under different load cases and environmental conditions. Exploration of faster yet still reliable methods to substitute for FEA in the optimum design procedure is therefore necessary. A number of rapid analysis methods have been embedded into layup optimization processes to counter the large number of iteration cycles which are always required. Todoroki and Ishikawa [3] used a response surface to obtain the buckling load with reduced computational cost. Hajmohammad et al. [4] employed an artificial neural network in their optimization to replace FEA when determining the buckling load of composite plates. An alternative to these methods is the exact finite strip approach for obtaining the buckling and postbuckling behaviour for prismatic plate assemblies [5-7]. The panel design package VICONOPT [8], based on the exact finite strip method, was used by Liu and Butler [9,10] to carry out optimum design of composite laminates. 
Over the past decades, genetic algorithms (GAs) have been the most popular optimization techniques for composite structures. Riche and Haftka [11] optimized stacking sequences for buckling load maximization using GAs. Park et al. [12] implemented GAs for maximum strength optimization of symmetric composite plates. In Almeida and Awruch's work [13] GAs were improved for optimizing a laminate's weight and deflection simultaneously. Chen et al. [14] used GAs with a two-level approximation in which the first level approximation was used for optimizing stacking sequences, and the related ply thicknesses were obtained during the second level approximation.

Due to the large number of design variables in optimization, lamination parameters which are independent of the number of layers were first introduced in [15] where they were used as intermediate design variables dividing the optimization into two separate stages. Yamazaki [16] used this two-stage optimization with lamination parameters as design variables in a gradient-based optimization in the first stage and searched stacking sequences with GAs to match those optimized lamination parameters in the second stage. When lamination parameters are used as design variables in layup optimization, their feasible regions are treated as constraints and have been studied in many works [17-21]. With this consideration, lamination parameters have been widely used in layup optimization [22-24].

When selecting optimal stacking sequences, the primary weakness of GAs is their high computational cost. For this reason, many alternative techniques have been explored. Bloomfield et al. [25] employed a particle swarm method to search the optimum stacking sequences in the second stage of their optimization. In later work [26] the selection of ply orientations using GAs, ant colony and particle swarm were studied and the methods compared, concluding the particle swarm techniques to be the best. Whilst good results could be obtained using ant colony and particle swam techniques, as meta-heuristic algorithms they still required high computational resources. Terada and Todoroki [27] used a fractal branch and bound method for stacking sequence optimization of flat plates, demonstrating large reductions in solution time. Further studies of the fractal branch and bound method were illustrated in their group's later work [28-32]. Narita and Turvey [33] employed a layerwise optimization approach to maximize the buckling load of a symmetric composite plate, and this approach achieved a good level of efficiency. A new logic-based branch and bound method combined with an improved global layerwise technique were explored in a two-stage optimization by Kennedy et al. [34], in which the exact strip analysis software VICONOPT was extended by introducing lamination parameters as design variables in the first stage. The logic-based method was used in the second stage to optimize the stacking sequence to match these optimized lamination parameters.

The authors' previous work [35] extended this logic-based method for the optimization of laminates which are required to be symmetric, balanced or both, under buckling and lamination parameter constraints. Manufacturing constraints, however, were not taken account into the optimization. In the present study, an improved two-stage optimization of composite laminates under buckling, in which manufacturing and further restricted lamination parameter constraints are implemented. During the first stage, the total laminate thickness and lamination parameters are optimized using VICONOPT in which the buckling constraints are satisfied by using a linear optimizer coupled with a stabilization technique [8], while the feasibility of the lamination parameters is ensured using a penalty function approach.

In the present work a new feasible region for the lamination parameters is obtained, when there is a minimum percentage requirement for plies with each of the four permissible orientations. During the second stage, four manufacturing constraints are added into the logic-based search, and the high efficiency of this method is illustrated by comparison with GA. Section 2 introduces the lamination parameters used in the two-stage optimization procedure, which is described in detail in section 3. Section 4 presents numerical results and solution times for a range of problems with different combinations of the manufacturing constraints, as well as comparisons with the results of other authors. Brief conclusions are given in section 5.

\section{Lamination parameters}

Classical laminate theory [1] presents the stress-strain relationship as

$$
\left[\begin{array}{l}
\mathbf{N} \\
\mathbf{M}
\end{array}\right]=\left[\begin{array}{ll}
\mathbf{A} & \mathbf{B} \\
\mathbf{B} & \mathbf{D}
\end{array}\right]\left[\begin{array}{l}
\boldsymbol{\varepsilon}^{0} \\
\mathbf{K}
\end{array}\right]
$$

where A, B and $\mathbf{D}$ are membrane, coupling and bending stiffness matrices, $\mathbf{N}$ and $\mathbf{M}$ are vectors of in-plane stresses and moments, $\boldsymbol{\varepsilon}^{0}$ is a vector of in-plane strains and $\boldsymbol{\kappa}$ is a vector of the mid plane curvatures. 
The stiffness matrices in Eq. (1) can be expressed in terms of material stiffness invariants $\mathbf{U}$ and 12 lamination parameters $\xi[19]$ as

$$
\begin{aligned}
& {\left[\begin{array}{l}
A_{11} \\
A_{22} \\
A_{12} \\
A_{66} \\
A_{16} \\
A_{26}
\end{array}\right]=h\left[\begin{array}{ccccc}
1 & \xi_{1}^{A} & \xi_{2}^{A} & 0 & 0 \\
1 & -\xi_{1}^{A} & \xi_{2}^{A} & 0 & 0 \\
0 & 0 & -\xi_{2}^{A} & 1 & 0 \\
0 & 0 & -\xi_{2}^{A} & 0 & 1 \\
0 & \xi_{3}^{A} / 2 & \xi_{4}^{A} & 0 & 0 \\
0 & \xi_{3}^{A} / 2 & -\xi_{4}^{A} & 0 & 0
\end{array}\right]\left[\begin{array}{l}
U_{1} \\
U_{2} \\
U_{3} \\
U_{4} \\
U_{5}
\end{array}\right]} \\
& {\left[\begin{array}{l}
B_{11} \\
B_{22} \\
B_{12} \\
B_{66} \\
B_{16} \\
B_{26}
\end{array}\right]=\frac{h^{2}}{4}\left[\begin{array}{ccccc}
0 & \xi_{1}^{B} & \xi_{2}^{B} & 0 & 0 \\
0 & -\xi_{1}^{B} & \xi_{2}^{B} & 0 & 0 \\
0 & 0 & -\xi_{2}^{B} & 0 & 0 \\
0 & 0 & -\xi_{2}^{B} & 0 & 0 \\
0 & \xi_{3}^{B} / 2 & \xi_{4}^{B} & 0 & 0 \\
0 & \xi_{3}^{B} / 2 & -\xi_{4}^{B} & 0 & 0
\end{array}\right]\left[\begin{array}{c}
U_{1} \\
U_{2} \\
U_{3} \\
U_{4} \\
U_{5}
\end{array}\right]} \\
& {\left[\begin{array}{l}
D_{11} \\
D_{22} \\
D_{12} \\
D_{66} \\
D_{16} \\
D_{26}
\end{array}\right]=\frac{h^{3}}{12}\left[\begin{array}{ccccc}
1 & \xi_{1}^{D} & \xi_{2}^{D} & 0 & 0 \\
1 & -\xi_{1}^{D} & \xi_{2}^{D} & 0 & 0 \\
0 & 0 & -\xi_{2}^{D} & 1 & 0 \\
0 & 0 & -\xi_{2}^{D} & 0 & 1 \\
0 & \xi_{3}^{D} / 2 & \xi_{4}^{D} & 0 & 0 \\
0 & \xi_{3}^{D} / 2 & -\xi_{4}^{D} & 0 & 0
\end{array}\right]\left[\begin{array}{l}
U_{1} \\
U_{2} \\
U_{3} \\
U_{4} \\
U_{5}
\end{array}\right]}
\end{aligned}
$$

The material stiffness invariants $\mathbf{U}$ and stiffness properties $\mathbf{Q}$ are presented as follows:

$$
\begin{gathered}
{\left[\begin{array}{l}
U_{1} \\
U_{2} \\
U_{3} \\
U_{4} \\
U_{5}
\end{array}\right]=1 / 8\left[\begin{array}{cccc}
3 & 2 & 3 & 4 \\
4 & 0 & -4 & 0 \\
1 & -2 & 1 & -4 \\
1 & 6 & 1 & -4 \\
1 & -2 & 1 & 4
\end{array}\right]\left[\begin{array}{l}
Q_{12} \\
Q_{12} \\
Q_{22} \\
Q_{66}
\end{array}\right]} \\
\left\{\begin{array}{l}
Q_{11}=E_{11}{ }^{2} /\left(E_{11}-E_{22} v_{12}{ }^{2}\right) \\
Q_{22}=E_{11} E_{22} /\left(E_{11}-E_{22} v_{12}{ }^{2}\right) \\
Q_{12}=v_{12} Q_{22} \\
Q_{66}=G_{12}
\end{array}\right.
\end{gathered}
$$

where $E_{11}$ is the longitudinal Young's modulus, $E_{22}$ is the transverse Young's modulus, $G_{12}$ is the shear modulus, $v_{12}$ is the major Poisson's ratio and $h$ is the thickness of the laminate.

The lamination parameters are calculated by the following integrals

$$
\left[\begin{array}{l}
\xi_{1}^{k} \\
\xi_{2}^{k} \\
\xi_{3}^{k} \\
\xi_{4}^{k}
\end{array}\right]=\int_{-h / 2}^{h / 2} Z^{k}\left[\begin{array}{l}
\cos 2 \theta \\
\cos 4 \theta \\
\sin 2 \theta \\
\sin 4 \theta
\end{array}\right] d z, k=A, B, D,\left\{\begin{array}{l}
Z^{A}=1 / h \\
Z^{B}=4 z / h^{2} \\
Z^{D}=12 z^{2} / h^{3}
\end{array}\right.
$$

where $\theta$ represents the ply orientation at depth $z$ below the mid-surface. If the ply orientations are limited to $0^{\circ}$, $90^{\circ},+45^{\circ}$ and $-45^{\circ}$, then $\xi_{4}^{k}(k=A, B, D)$ are zero. The coupling lamination parameters $\xi_{i}^{B}(i=1,2,3,4)$ are zero when the laminate is symmetric. Also, $\xi_{3}^{A}$ is zero for a balanced laminate. 


\section{Optimization procedure}

The aim of the optimization is to minimize the mass of the composite laminate under a compressive load. The constraints are the buckling, manufacturing and lamination parameter constraints. The optimization approach is divided into two stages. The lamination parameters and total laminate thickness are used as design variables in the first stage. Ply orientations, which are restricted to $0^{\circ}, 90^{\circ},+45^{\circ}$ and $-45^{\circ}$ are used to build the actual layup for the laminate in the second stage.

\subsection{Manufacturing constraints}

Niu [36] listed manufacturing constraints for the design of aircraft composite materials. In this paper, four major manufacturing constraints for composite laminate design are taken into account in a two-stage optimization process, as follows.

(1) Contiguity constraint: the maximum number of successive plies with the same orientation is limited to an integer $n_{\text {cont }}$ to minimize edge splitting. $n_{\text {cont }}=4$ in this paper.

(2) Disorientation constraint: the difference between two adjacent plies should be less than an angle $\theta_{\text {diff }}$. This constraint is applied to avoid microcracking. $\theta_{\text {diff }}=60^{\circ}$ in this paper.

(3) Minimum percentage constraint: each fiber orientation should comprise a proportion of at least $p_{m}$ of the total layup to prevent the matrix from being exposed to direct loads and provide sufficient damage tolerance to the laminate. $p_{\min }=10 \%$ in this paper.

(4) Damage tolerance (damtol) constraint: avoid putting $0^{\circ}$ and $90^{\circ}$ plies on the exterior surfaces of the laminate to provide sufficient damage tolerance after impact.

\subsection{First stage optimization}

The exact strip software VICONOPT which comprises the earlier programs VIPASA [5] (Vibration and Instability of Plate Assemblies including Shear and Anisotropy) and VICON [6] (VIPASA with CONstraints) performs buckling, postbuckling and free vibration analyses of prismatic plate assemblies based on the Wittrick-Williams algorithm [5], and provides a strong structural optimization tool in preliminary aircraft design. The major advantage of VICONOPT over FEA is its high efficiency, being typically 1000 or 150 times faster for buckling analyses using its VIPASA and VICON options, respectively [8]. For continuous optimization problems, buckling constraints are evaluated by exact strip eigenvalue analysis, and their sensitivities with respect to all the design variables are calculated using an accelerated finite difference technique [8]. Next, assuming local linear approximations for the buckling constraints, the design variables are changed iteratively using the method of feasible directions [37]. A thickness factoring technique [8] is then applied to obtain a just stable configuration.

Recently, the optimization capability of VICONOPT has been expanded by introducing lamination parameters as design variables, so that the optimization problem in the first stage can be expressed as:

$$
\begin{aligned}
& \text { Minimize } M(\boldsymbol{x}) \\
& \text { Subject to } G_{i}(\boldsymbol{x}) \leq 0 \quad i=1,2,3, \ldots, m \\
& x_{j}^{l} \leq x_{j} \leq x_{j}^{u} \quad j=1,2,3, \ldots, n(n \leq 10)
\end{aligned}
$$

where $M(x)$ is the laminate mass. $G_{i}(x)$ are inequality design constraints, including buckling and lamination parameter constraints. The minimum percentage constraint, i.e. manufacturing constraint ( 3 ) above, is added to the lamination parameter constraints in this paper. $\boldsymbol{x}=\left(h, \xi_{1}^{A}, \xi_{2}^{A}, \xi_{3}^{A}, \xi_{1}^{D}, \xi_{2}^{D}, \xi_{3}^{D}, \xi_{1}^{B}, \xi_{2}^{B}, \xi_{3}^{B}\right)$ is the vector of design variables, $m$ is the number of constraints and $n$ is the number of design variables.

The following 25 non-linear lamination parameter constraints are applied [18, 19,34], giving a restricted feasible region.

$$
\begin{gathered}
2\left|\xi_{1}^{k}\right|-\xi_{2}^{k}-1 \leq 0, \quad 2\left|\xi_{3}^{k}\right|+\xi_{2}^{k}-1 \leq 0 \quad k=A, D \\
2\left|\xi_{1}^{B}\right|+\xi_{2}^{B}-2 \leq 0, \quad 2\left|\xi_{3}^{B}\right|+\xi_{2}^{B}-2 \leq 0, \quad\left|\xi_{1}^{B}\right|+\left|\xi_{3}^{B}\right|-1 \leq 0 \\
4\left(\xi_{i}^{D}+r\right)\left(\xi_{i}^{A}+r\right) \geq\left(\xi_{i}^{A}+r\right)^{4}+3\left(\xi_{i}^{B}\right)^{2}, \quad i=1,2,3, \quad r= \pm 1
\end{gathered}
$$




$$
\begin{gathered}
16\left(2 \xi_{1}^{D}+r \xi_{2}^{D}+r s\right)\left(2 \xi_{1}^{A}+r \xi_{2}^{A}+r s\right) \geq\left(2 \xi_{1}^{A}+r \xi_{2}^{A}+r s\right)^{4}+12\left(2 \xi_{1}^{B}+r \xi_{2}^{B}\right)^{2}, \\
r= \pm 1, \quad s=1,-3 \\
16\left(2 \xi_{3}^{D}+r \xi_{2}^{D}+r s\right)\left(2 \xi_{3}^{A}+r \xi_{2}^{A}+r s\right) \geq\left(2 \xi_{3}^{A}+r \xi_{2}^{A}+r s\right)^{4}+12\left(2 \xi_{3}^{B}+r \xi_{2}^{B}\right)^{2}, \\
r= \pm 1, \quad s=1,-3 \\
4\left(\xi_{1}^{D}+r \xi_{3}^{D}+s\right)\left(\xi_{1}^{A}+r \xi_{3}^{A}+s\right) \geq\left(\xi_{1}^{A}+r \xi_{3}^{A}+s\right)^{4}+3\left(\xi_{1}^{B}+r \xi_{3}^{B}\right)^{2}, r= \pm 1, \quad s= \pm 1
\end{gathered}
$$

The lamination parameter constraints of Equations (8-13) are implemented by using a penalty function method. It is noted that the feasible region is convex [18] and that the constraints of Equations (8-13) are satisfied if all the lamination parameters $\xi_{j}^{k}$ are set to zero. Thus when any of the constraints is violated, all the lamination parameters $\xi_{j}^{k}$ and laminate thicknesses $h$ are multiplied by a scalar $\alpha(0<\alpha<1)$ so that the most critical constraint is just satisfied, converting an infeasible configuration to an artificial just feasible one. In the stiffness matrix calculations, reducing the thickness $h$ to $\alpha h$ makes the infeasible configuration unattractive by giving it an artificially low stiffness which tends to violate the buckling constraints. However, the laminate thickness $h$ is not reduced in the mass calculations so the infeasible configuration will not have an artificially attractive objective function. Details of this process can be found in [34].

When giving the percentage of each ply orientation a minimum value $p_{\min }$, the feasible region of the lamination parameters is further restricted. Because the number of plies of each orientation is directly related to the in-plane lamination parameters $\xi_{1,2,3}^{A}$, and the contribution of each angle is known as shown in Table 1, the relationship between the number of plies with each orientation and the three lamination parameters $\xi_{1,2,3}^{A}$ can be expressed as follows.

$$
\begin{gathered}
n_{0}+n_{45}+n_{-45}+n_{90}=n \\
n_{0}-n_{90}=n \xi_{1}^{A} \\
n_{0}-n_{45}-n_{-45}+n_{90}=n \xi_{2}^{A} \\
n_{45}-n_{-45}=n \xi_{3}^{A}
\end{gathered}
$$

where the non-negative integers $n$ and $n_{\theta}$ represent the total number of plies and the number of plies with orientation $\theta$, respectively. The minimum percentage constraint can be expressed as

$$
\begin{gathered}
n_{0} / n=\xi_{1}^{A} / 2+\xi_{2}^{A} / 4+1 / 4 \geq p_{\min } \\
n_{90} / n=-\xi_{1}^{A} / 2+\xi_{2}^{A} / 4+1 / 4 \geq p_{\min } \\
n_{45} / n=-\xi_{2}^{A} / 4+\xi_{3}^{A} / 2+1 / 4 \geq p_{\min } \\
n_{-45} / n=-\xi_{2}^{A} / 4-\xi_{3}^{A} / 2+1 / 4 \geq p_{\min }
\end{gathered}
$$

The related lamination parameter constraints are then obtained as

$$
2\left|\xi_{1}^{A}\right|-\xi_{2}^{A}+4 p_{\min }-1 \leq 0, \quad 2\left|\xi_{3}^{A}\right|+\xi_{2}^{A}+4 p_{\min }-1 \leq 0
$$

Table 1

Contribution of each ply orientation to the in-plane lamination parameters.

\begin{tabular}{cccc}
\hline & $\xi_{1}^{A}$ & $\xi_{2}^{A}$ & $\xi_{3}^{A}$ \\
\hline $0^{\circ}$ & 1 & 1 & 0 \\
$+45^{\circ}$ & 0 & -1 & 1 \\
$-45^{\circ}$ & 0 & -1 & -1 \\
$90^{\circ}$ & -1 & 1 & 0 \\
\hline
\end{tabular}


Hence, $\xi_{2}^{A} \in\left[4 p_{\min }-1,1-4 p_{\min }\right]$. Because of the relationship between $\xi_{1,2,3}^{A}$ and $\xi_{1,2,3}^{D}$ as illustrated in equation (10), $\xi_{2}^{D} \in\left[16 p_{\min ^{3}}{ }^{3}-1,1-16 p_{\min }{ }^{3}\right]$. Therefore, the corresponding relationship between $\xi_{1,2,3}^{D}$ for symmetric laminate (i.e. $\xi_{1,2,3}^{B}=0$ ) are changed as

$$
2\left|\xi_{1}^{D}\right|-\xi_{2}^{D}+16 p_{\min ^{3}}{ }^{3}-1 \leq 0,2\left|\xi_{3}^{D}\right|+\xi_{2}^{D}+16 p_{\min }{ }^{3}-1 \leq 0
$$

Feasible regions for $\xi_{1,2,3}^{A}$ and $\xi_{1,2,3}^{D}$ are shown in Figs. 1 and 2, respectively. The triangular regions with the dashed lines represent the previous feasible regions given by equation (8). In this paper, $p_{\min }$ is required to be 0.1 and when the minimum percentage constraint is added, these triangles are reduced to the smaller triangles surrounded by the solid lines. It is seen that the area of the feasible regions of $\xi_{1,2,3}^{A}$ is reduced by $64 \%$ while those of $\xi_{1,2,3}^{D}$ are reduced by only about $3 \%$.
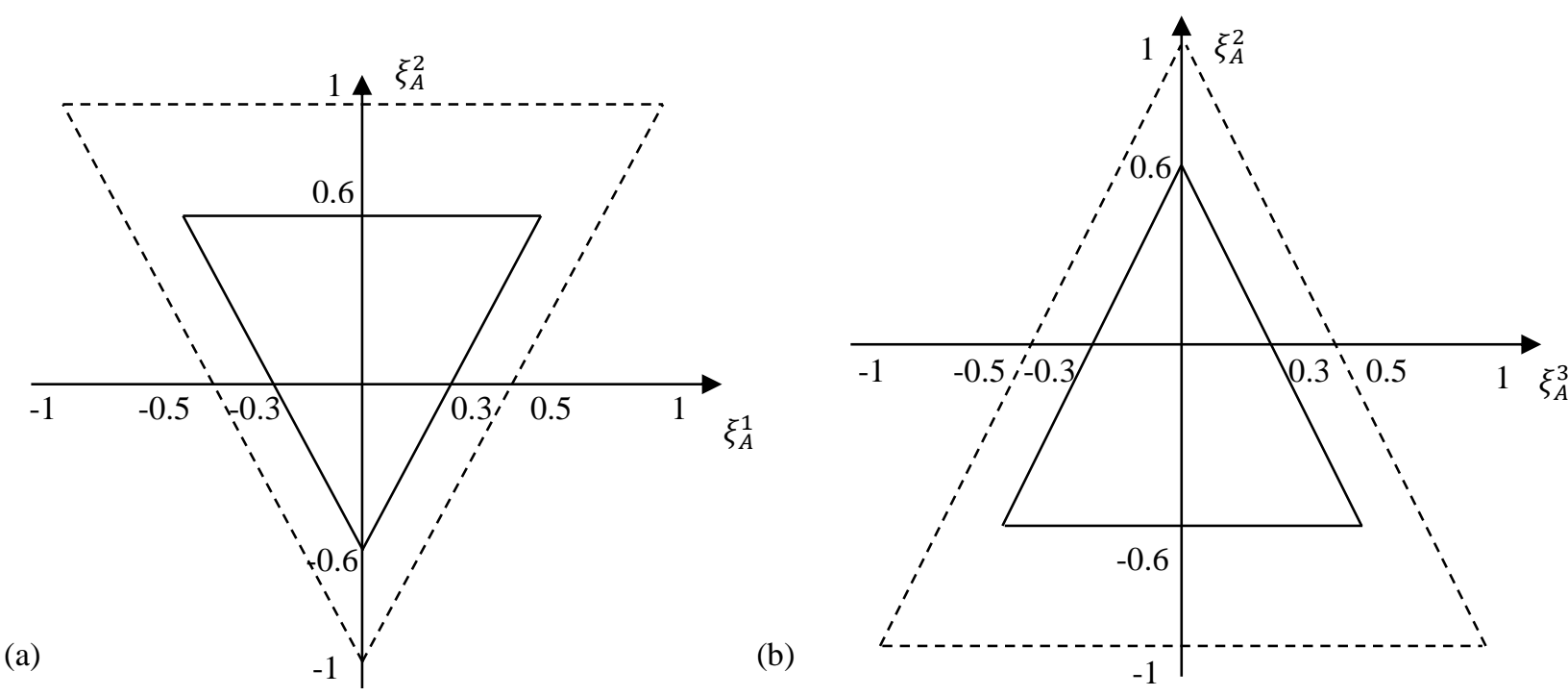

Fig. 1. Restricted feasible regions of lamination parameters $\xi_{1,2,3}^{A}$, when considering the minimum percentage constraint $p_{\min }=0.1$.
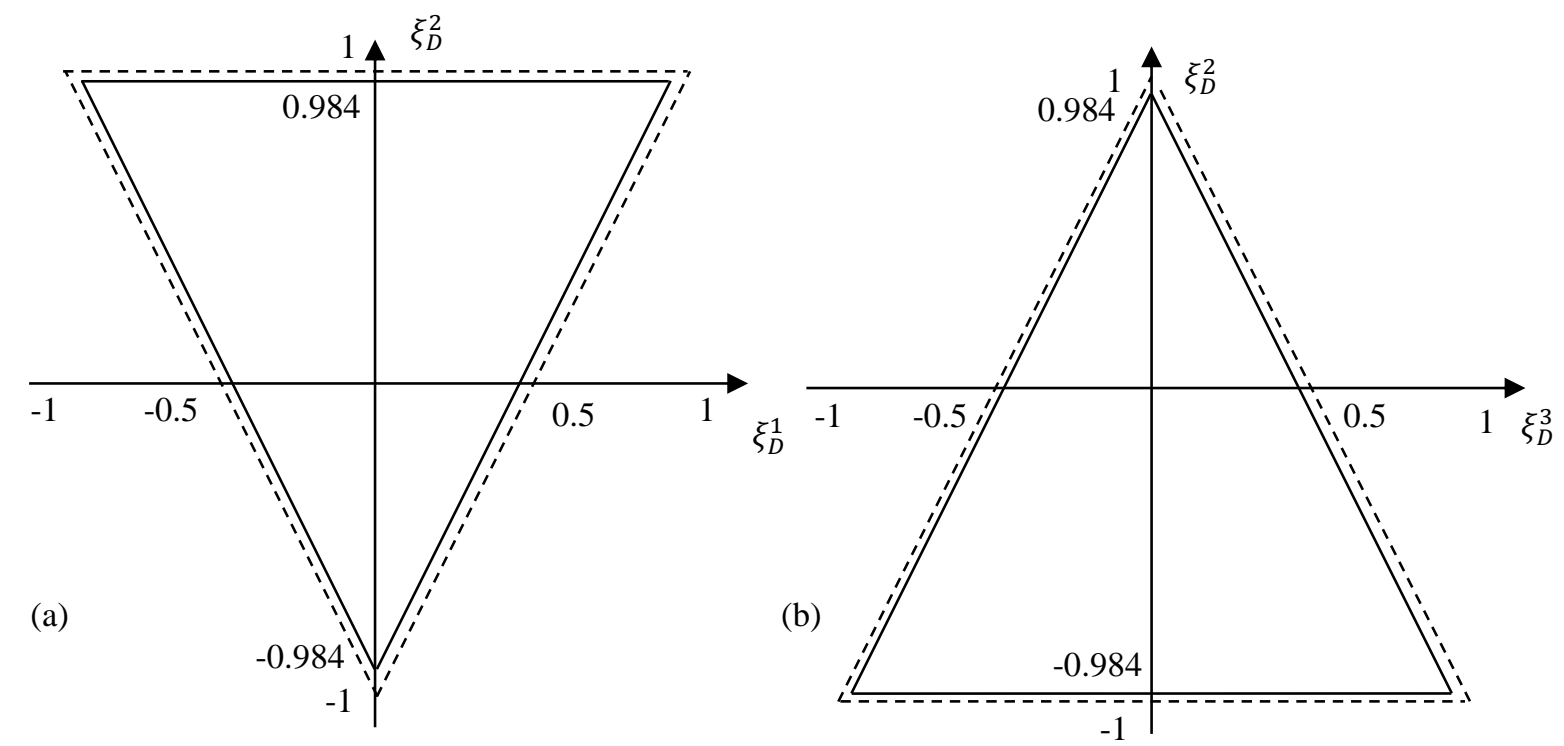

Fig. 2. Restricted feasible regions of lamination parameters $\xi_{1,2,3}^{D}$, for symmetric laminate, when considering the minimum percentage constraint $p_{\min }=0.1$. 


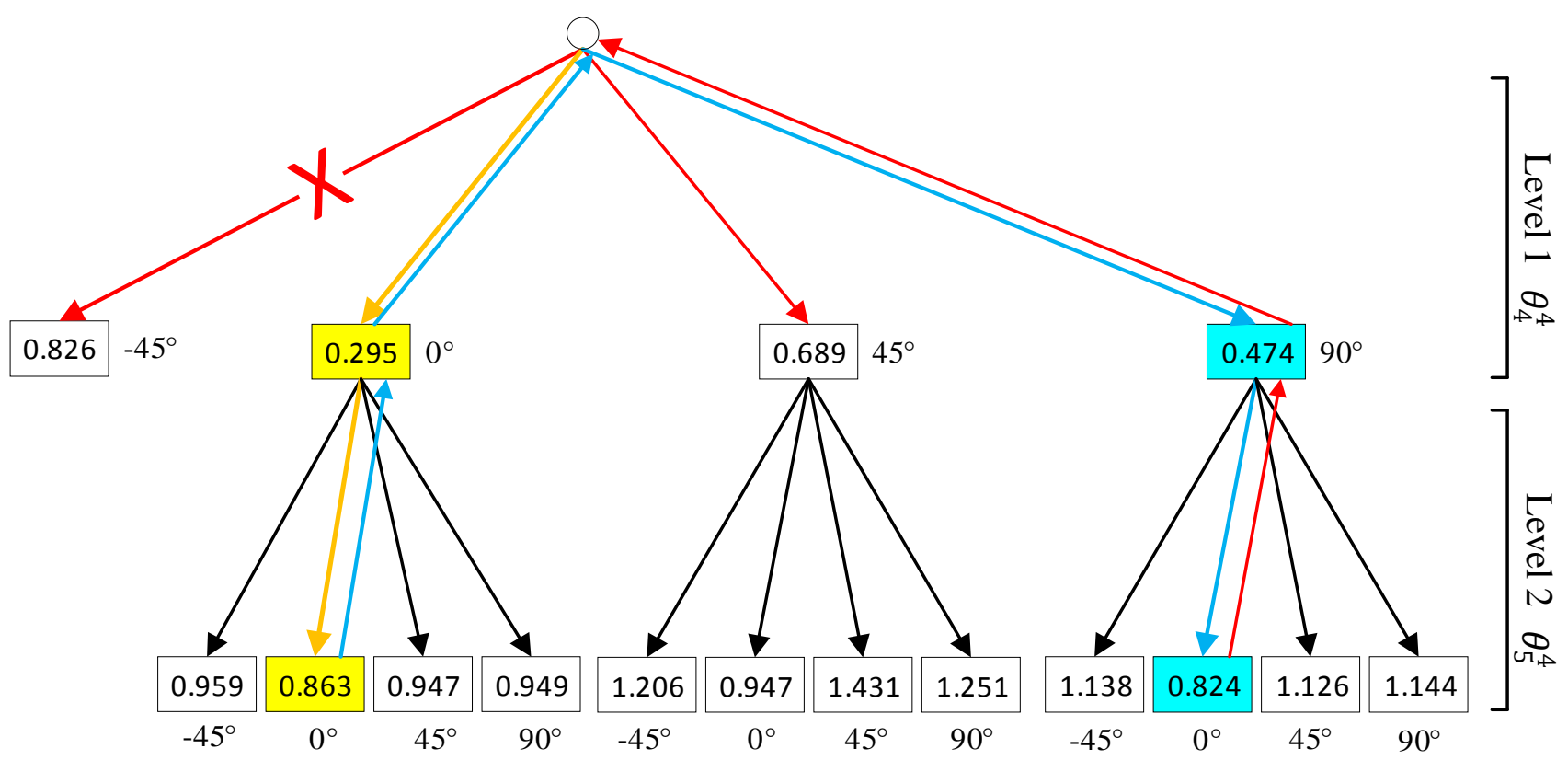

Fig. 3. An illustrative branch and bound decision tree for optimizing two plies.

\subsection{Second stage optimization}

After the first stage, the optimized laminate thickness $h$ is rounded to the nearest integer multiple $n h_{p}$ of the ply thickness $h_{p}$, and the optimized lamination parameters are used as target values in the second stage. The aim of this second stage is a layup to match the target values as closely as possible, with logic-based methods and the most popular technique GAs both having this ability to search the layup.

\subsubsection{Logic-based method}

The logic-based method is a combination of the branch and bound method and a global layerwise technique. In the branch and bound method, branches (i.e. choice options) constitute the decision tree of which the size (i.e. the number of level) is dependent on the numbers of plies to be optimized. The structure of a decision tree which only considers two plies is shown in Fig. 3. As the problem size increases, the search time tends to grow dramatically, for example a decision tree with 16 levels potentially has $4^{16}$ possibilities to search. Therefore, the global layerwise technique is developed to improve the efficiency of the branch and bound process. The objective function $\Gamma$ is obtained by calculating the difference between the target lamination parameters given by VICONOPT and the actual lamination parameters related to the chosen ply orientations as follows.

$$
\Gamma=\sum_{i=1}^{3} \sum_{j=A}^{A, B, D} w_{j}\left|\xi_{i(\text { actual })}^{j}-\xi_{i(\text { target })}^{j}\right|
$$

where $w_{A, B, D}$ are weighting factors, $\xi_{1,2,3 \text { (target) }}^{A, B, D}$ are the target lamination parameters obtained in the first stage, and $\xi_{1,2,3 \text { (actual) }}^{A, B, D}$ are the actual lamination parameters for the chosen layup.

The branching process can predict the route to proceed with in the next level of the decision tree by considering bounds on the achievable values of the objective function. The aim is to opt for a branch which is close to the target value of the lamination parameters. If $\Gamma$ is lower than that of the previous best solution, the chosen layup is saved as the incumbent solution. The bounding process is used to discard the branches which cannot improve on the objective function of the incumbent solution or which violate constraints (e.g. manufacturing constraints, balanced constraint, symmetric constraint). Thus the process operates most efficiently on small problems or when there is a good incumbent solution.

To obtain good incumbent solutions as quickly as possible in the process, the global layerwise technique is employed to accelerate the search. This technique has 3 nested loops: cycle, pass and case, as shown in Figs. 4 and 5. The process is illustrated in Fig. 4 using an example of an 8 ply laminate. For each case, the plies available 
for selection by the branch and bound method are shown in bold, and the optimization starts from an arbitrary layup $\left(\theta_{1}^{0}, \theta_{2}^{0}, \theta_{3}^{0}, \theta_{4}^{0}, \theta_{5}^{0}, \theta_{6}^{0}, \theta_{7}^{0}, \theta_{8}^{0}\right)$. The ply orientations are optimized successively, working inwards from the outer plies which make the most important contributions to the out-of-plane lamination parameters $\xi_{1,2,3}^{D}$. In the first cycle, two plies are optimized at once. In the first case of the first pass of the first cycle the two outermost plies are optimized to become $\left(\theta_{1}^{1}, \theta_{8}^{1}\right)$. The newly optimized layup is then used as starting layup for the second case where the solution is $\left(\theta_{2}^{1}, \theta_{7}^{1}\right)$, and so on. When the innermost plies $\left(\theta_{4}^{1}, \theta_{5}^{1}\right)$ are obtained, the first pass of the first cycle is completed and a second pass of the first cycle is made until no further changes are made. In the second cycle, 4 plies are optimized at once, again with the process being repeated until the value of $\Gamma$ cannot be reduced. The number of variable plies in the third cycle is 6 (or fewer), while all the plies are optimized in the final cycle. (For brevity in Fig. 4, two passes are shown in cycle 2, but only one pass for the other cycles.) Therefore, the branch and bound method initially optimizes the layup by searching a small decision tree meaning that very low values of $\Gamma$ can be obtained quickly during the first few cycles. Subsequently, when searching lager numbers of plies, the previous incumbent value of $\Gamma$ is used in the bounding process, enabling many branches to be discarded without being explored. Therefore, optimized stacking sequences can be obtained efficiently.

During each case, after a new incumbent layup has been chosen or it has been shown that the currently chosen branch cannot improve on the objective function no matter which lower branches are chosen, the remaining branches in the decision tree must subsequently be explored (to seek better solutions) or discarded (if they cannot improve on the incumbent solution) in order to prove global optimality. An example is given to illustrate this backtracking process. Suppose the branch and bound process is working on the fourth case of the first pass of the first cycle as shown in Fig. 4, of which the decision tree is shown in Fig. 3. The required lamination parameters are $\xi_{1}^{A}=0.27, \xi_{2}^{A}=0.2, \xi_{3}^{A}=-0.08, \xi_{1}^{D}=-0.09, \xi_{2}^{D}=-0.12, \xi_{3}^{D}=-0.44, \xi_{1}^{B}=0.1, \xi_{2}^{B}=0.8, \xi_{3}^{B}=0.1$, and the incumbent layup before this case is $[-45 / 45 /-45 / 90 / 90 / 0 / 0 / 90]$ with the value of $\Gamma=1.144$. The numbers in the boxes at the lowest level of the decision tree are the exact values of $\Gamma$ for the complete layup. For those at the first level, because the layup has not yet been completed, the numbers are lower bounds for each branch, which are obtained by subtracting the maximum achievable contribution of the remaining levels from the exact value of $\Gamma$ obtained from the contributions of the preceding levels. For example, for the branch $0^{\circ}$ at the first level in Fig. 3 , the ply angle at the second level has not been chosen and the provisional value of $\Gamma$ for the uncompleted layup [-45/45/-45/0/\#/0/0/90] is 0.787 . The maximum achievable contribution of each of the lamination parameters of the ply at the second level can be obtained by the following equation

$$
\left[\begin{array}{l}
\xi_{1}^{k} \\
\xi_{2}^{k} \\
\xi_{3}^{k}
\end{array}\right]=\left|\int_{-h / 8}^{0} Z^{k} d z\right|, k=A, B, D,\left\{\begin{array}{l}
Z^{A}=1 / h \\
Z^{B}=4 z / h^{2} \\
Z^{D}=12 z^{2} / h^{3}
\end{array}\right.
$$

\begin{tabular}{|c|c|c|c|c|c|c|c|c|c|c|c|c|}
\hline Cycle & 0 & 1 & 1 & 1 & 1 & 2 & 2 & 2 & 2 & 3 & 3 & 4 \\
\hline Pass & 0 & 1 & 1 & 1 & 1 & 1 & 1 & 2 & 2 & 1 & 1 & 1 \\
\hline Case & 0 & 1 & 2 & 3 & 4 & 5 & 6 & 7 & 8 & 9 & 10 & 11 \\
\hline Var. plies & 2 & 2 & 2 & 2 & 2 & 4 & 4 & 4 & 4 & 6 & 2 & 8 \\
\hline Ply1 & $\theta_{1}^{0}$ & $\theta_{1}^{1}$ & $\theta_{1}^{1}$ & $\theta_{1}^{1}$ & $\theta_{1}^{1}$ & $\theta_{1}^{5}$ & $\theta_{1}^{5}$ & $\theta_{1}^{7}$ & $\theta_{1}^{7}$ & $\theta_{1}^{9}$ & $\theta_{1}^{9}$ & $\theta_{1}^{11}$ \\
\hline Ply2 & $\theta_{2}^{0}$ & $\theta_{2}^{0}$ & $\theta_{2}^{2}$ & $\theta_{2}^{2}$ & $\theta_{2}^{2}$ & $\theta_{2}^{5}$ & $\theta_{2}^{5}$ & $\theta_{2}^{7}$ & $\theta_{2}^{7}$ & $\theta_{2}^{9}$ & $\theta_{2}^{9}$ & $\theta_{2}^{11}$ \\
\hline Ply3 & $\theta_{3}^{0}$ & $\theta_{3}^{0}$ & $\theta_{3}^{0}$ & $\boldsymbol{\theta}_{3}^{3}$ & $\theta_{3}^{3}$ & $\theta_{3}^{3}$ & $\theta_{3}^{6}$ & $\theta_{3}^{6}$ & $\boldsymbol{\theta}_{3}^{\mathbf{8}}$ & $\theta_{3}^{9}$ & $\theta_{3}^{9}$ & $\theta_{3}^{11}$ \\
\hline Ply4 & $\theta_{4}^{0}$ & $\theta_{4}^{0}$ & $\theta_{4}^{0}$ & $\theta_{4}^{0}$ & $\theta_{4}^{4}$ & $\theta_{4}^{4}$ & $\theta_{4}^{6}$ & $\theta_{4}^{6}$ & $\theta_{4}^{8}$ & $\theta_{4}^{8}$ & $\theta_{4}^{10}$ & $\theta_{4}^{11}$ \\
\hline Ply5 & $\theta_{5}^{0}$ & $\theta_{5}^{0}$ & $\theta_{5}^{0}$ & $\theta_{5}^{0}$ & $\theta_{5}^{4}$ & $\theta_{5}^{4}$ & $\theta_{5}^{6}$ & $\theta_{5}^{6}$ & $\theta_{5}^{8}$ & $\theta_{5}^{8}$ & $\theta_{5}^{10}$ & $\theta_{5}^{11}$ \\
\hline Ply6 & $\theta_{6}^{0}$ & $\theta_{6}^{0}$ & $\theta_{6}^{0}$ & $\theta_{6}^{3}$ & $\theta_{6}^{3}$ & $\theta_{6}^{3}$ & $\theta_{6}^{6}$ & $\theta_{6}^{6}$ & $\theta_{6}^{8}$ & $\theta_{6}^{9}$ & $\theta_{6}^{9}$ & $\theta_{6}^{11}$ \\
\hline Ply7 & $\theta_{7}^{0}$ & $\theta_{7}^{0}$ & $\theta_{7}^{2}$ & $\theta_{7}^{2}$ & $\theta_{7}^{2}$ & $\theta_{7}^{5}$ & $\theta_{7}^{5}$ & $\theta_{7}^{7}$ & $\theta_{7}^{7}$ & $\theta_{7}^{9}$ & $\theta_{7}^{9}$ & $\theta_{7}^{11}$ \\
\hline Ply8 & $\theta_{8}^{0}$ & $\theta_{8}^{1}$ & $\theta_{8}^{1}$ & $\theta_{8}^{1}$ & $\theta_{8}^{1}$ & $\boldsymbol{\theta}_{8}^{5}$ & $\theta_{8}^{5}$ & $\theta_{8}^{7}$ & $\theta_{8}^{7}$ & $\theta_{8}^{9}$ & $\theta_{8}^{9}$ & $\theta_{8}^{11}$ \\
\hline
\end{tabular}

Fig. 4. Application of the global layerwise technique to optimization of an 8 ply laminate. 


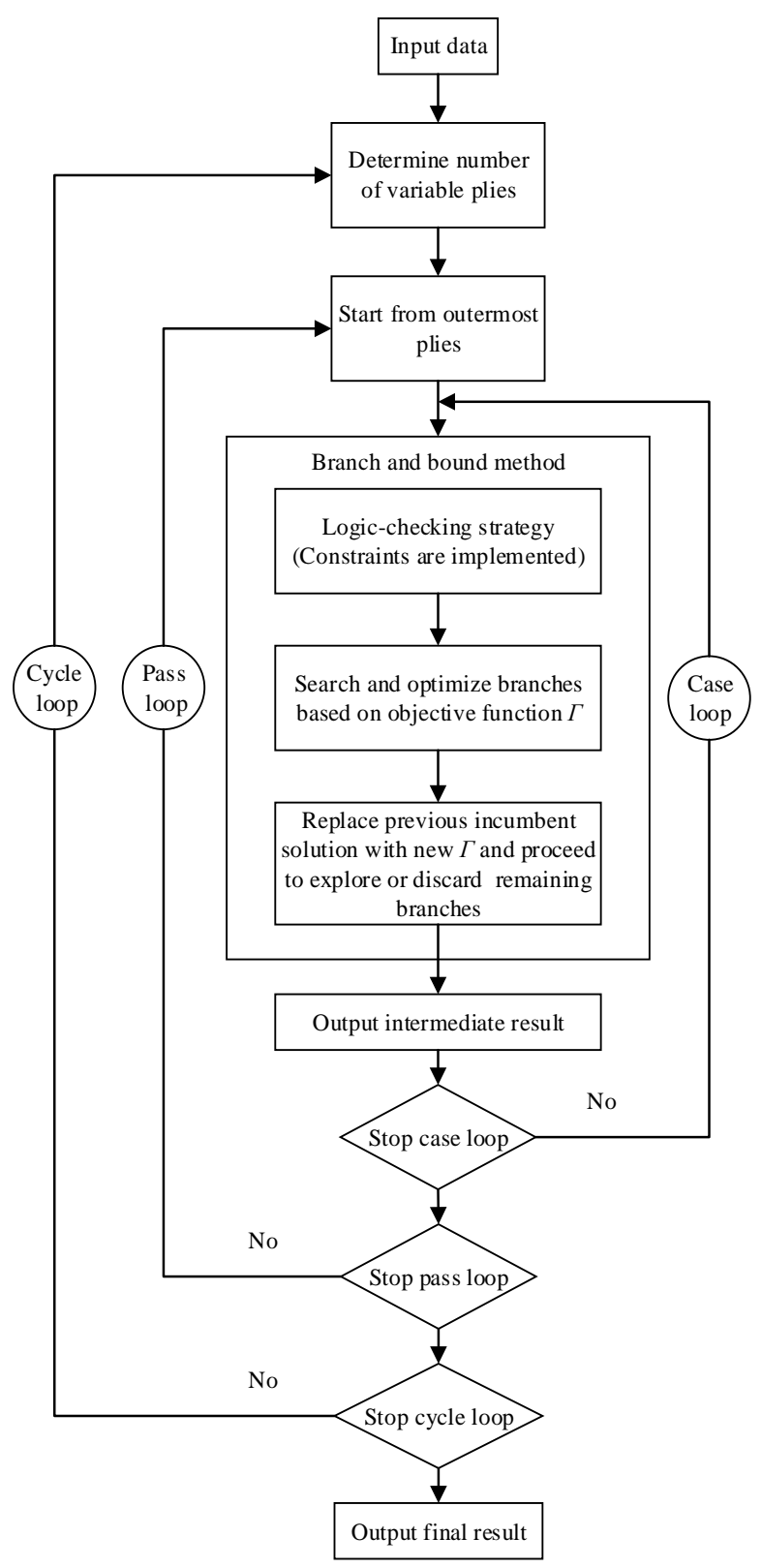

Fig. 5. Flow chart of logic-based method.

Hence, the maximum achievable contributions of the ply at the second level are summed to 0.492 , and the lower bound for the $0^{\circ}$ branch at the first level is obtained as $0.787-0.492=0.295$. Actually, this lower bound cannot be reached because the maximum contributions of each lamination parameter cannot be achieved simultaneously, but it can be used as a conservative limit. As can be seen from the decision tree, the branch $0^{\circ}$ (whose lower bound 0.295 is the lowest) is chosen at the first level in the initial search (see the yellow arrows and boxes). Then at the second level the best layup $0^{\circ} / 0^{\circ}$ is chosen, and the incumbent value of $\Gamma$ decreases to 0.863 which is used as a new upper bound in the process. Then the backtracking process starts to check the remaining branches. The second best branch $90^{\circ}$ at the first level is explored first (see the blue arrows and boxes), and a better layup $90^{\circ} / 0^{\circ}$ is then found at the second level enabling the incumbent value of $\Gamma$ to be decreased to 0.824 . This is lower than the lower bound 0.826 of branch $-45^{\circ}$ at the first level, indicating that better incumbent solutions cannot be found on this branch no matter which lower branches are chosen. Hence it can be discarded directly (see the red arrows) without being explored further, thus improving the efficiency of the process. Finally the backtracking process completes searching the branch $45^{\circ}$ and its lower branches without finding a better layup, so that the layup $90^{\circ} / 0^{\circ}$ is proved to be the global optimum in the current case.

Compared with stochastic search methods, the method presented herein is more reliable for finding a global optimum, because it logically searches all the possibilities of the stacking sequence. Often optimal results can be 
obtained at an early stage even for the panels with large number of plies. However, it takes a long time to prove optimality for panels with a large number of plies since the decision trees in later cycles are very large. Therefore, on large problems it is recommended to apply a stopping criterion such as an acceptably low value of $\Gamma$ (e.g. 0.2 ), maximum solution time or the completion of a specific cycle (e.g. 9th cycle)), after which the incumbent solution will be accepted without proving optimality.

\subsubsection{Addition of constraints}

A logic-based checking strategy is used for implementing constraints in the search process. When adding constraints such as the symmetric constraint, the balanced constraint and the manufacturing constraints in the optimization, this strategy checks and discards any branches violating constraints before every branching decision is made. Therefore, only the branches satisfying the constraints are searched at each level of the decision tree, ensuring that all the intermediate results satisfy the required constraints.

When the layup is required to be symmetric, the branching process is used to find the orientation of each ply in the top half of the laminate, and then the checking strategy will force the symmetrically located ply in the bottom half to have the same orientation, essentially halving the number of levels in the decision tree.

The balanced constraint is implemented by checking the difference between the number of plies which have already been chosen with $+45^{\circ}\left(n_{45}\right)$ and those with $-45^{\circ}\left(n_{-45}\right)$ at every level of the decision tree. If the value of $n_{45}-n_{-45}$ is equal to $n_{\text {left }}$, the number of plies left to choose in the current case, all the remaining plies must be set to $-45^{\circ}$. If the value $n_{45}-n_{-45}=n_{\text {left }}-1$, the next ply cannot be set to $+45^{\circ}$. Analogous rules apply for $-45^{\circ}$. As shown in the examples in Fig. 6 , if there are 8 plies in a case and the first 6 plies have already been chosen, $\theta_{4}$ is under selection. In Fig. $6(\mathrm{a})$, two $+45^{\circ}$ plies and one $-45^{\circ}$ ply have been chosen and there are only two plies left, so $\theta_{4}$ is not allowed to be $+45^{\circ}$. In Fig. 6 (b) $\theta_{4}$ must be set to $-45^{\circ}$, owing to the fact that there are already three $-45^{\circ}$ plies but only one $+45^{\circ}$ ply with two plies left to choose. This procedure has advantages over a commonly used alternative strategy which combines a $+45^{\circ}$ ply with a $-45^{\circ}$ ply as a single design variable, which automatically violating the disorientation constraint, i.e. manufacturing constraint (2) above, and potentially misses the global optimal result. Nevertheless, all possible balanced stacking sequences are searched using this logic-based strategy, so the global optimal layup under the balanced constraint can be achieved.

Four manufacturing constraints are included in the logic-based search through the logic-checking strategy. The contiguity constraint is implemented by checking plies which would constitute an adjacent group with more than $n_{\text {cont }}$ identical orientations. If such a group is formed the bounding process is forced to discard this choice.

When considering the disorientation constraint, the logic-based strategy checks the already chosen plies which are adjacent to the ply under selection, and discards the choices which would makes the difference with adjacent

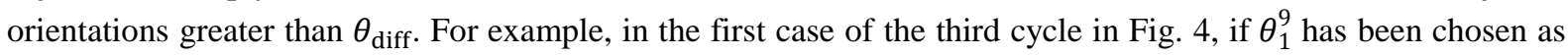
$+45^{\circ}$, ply 2 is under consideration, and ply 3 has not yet been chosen, the choices for ply 2 are compared with $\theta_{1}^{9}$ and are restricted to $0^{\circ},+45^{\circ}, 90^{\circ}$, i.e. the choice $\theta_{2}^{9}=-45^{\circ}$ is discarded. Suppose $\theta_{2}^{9}$ is chosen to be $0^{\circ}$. Then, when ply 3 is being chosen it is necessary to consider $\theta_{2}^{9}$ and $\theta_{4}^{8}$ simultaneously because $\theta_{4}^{8}$ was chosen in the previous case and is fixed in this case. So if $\theta_{4}^{8}$ is $90^{\circ}, \theta_{3}^{9}$ can only become $-45^{\circ}$ or $+45^{\circ}$.

\begin{tabular}{|c|}
\hline$\theta_{1}\left(0^{\circ}\right)$ \\
\hline$\theta_{2}\left(45^{\circ}\right)$ \\
\hline$\theta_{3}\left(90^{\circ}\right)$ \\
\hline$\Longrightarrow \theta_{4}$ \\
\hline$\theta_{5}$ \\
\hline$\theta_{6}\left(-45^{\circ}\right)$ \\
\hline$\theta_{7}\left(0^{\circ}\right)$ \\
\hline$\theta_{8}\left(45^{\circ}\right)$ \\
\hline
\end{tabular}

(a)

\begin{tabular}{|c|}
\hline$\theta_{1}\left(-45^{\circ}\right)$ \\
\hline$\theta_{2}\left(0^{\circ}\right)$ \\
\hline$\theta_{3}\left(45^{\circ}\right)$ \\
\hline$\theta_{4}$ \\
\hline$\theta_{5}$ \\
\hline$\theta_{6}\left(-45^{\circ}\right)$ \\
\hline$\theta_{7}\left(-45^{\circ}\right)$ \\
\hline$\theta_{8}\left(90^{\circ}\right)$ \\
\hline
\end{tabular}

(b)

Fig. 6. Checking strategy for balanced constraint. (a) $\theta_{4}$ cannot be $+45^{\circ}$. (b) $\theta_{4}$ must be $+45^{\circ}$. 
As for the minimum percentage constraint, this has been included with the lamination parameters constraints in the first stage. However, to avoid a rare situation where the lamination parameters of the actual layup are located just outside of the feasible region, the minimum percentage constraint is also included in the logic-based search. During the process, the percentage of each angle is calculated every time a new ply orientation is chosen, and results violating this constraint are abandoned.

The damtol constraint is implemented by discarding the $0^{\circ}$ and $90^{\circ}$ branches when processing with the two outermost plies.

The initial layup for this logic-based method is normally chosen as a multiple of a ply group of [-45/0/45/90]s, which can constitute laminates with number of plies equal to multiples of eight. For laminates with other numbers of plies, combinations of the following three groups [-45/45]s, [90 or 0]s, [0] are added at the middle of the laminate. Note that these plies should be placed next to plies with same angle to avoid violating the disorientation constraint. Thus the logic-based search starts with a layup which satisfies all the constraints, ensuring that all the intermediate results and final result satisfy these constraints.

\subsubsection{Genetic Algorithm}

As the most popular method in layup optimization, GAs based on a stochastic search have also been employed in the second stage as an alternative to the logic-based method in order to make a comparison of the two techniques. The GA used in this paper is composed of the following procedures:

1. Generate an initial population of random layups.

2. Select individuals for reproduction based on a fitness function.

3. Create a new generation through crossover, mutation and permutation [11] operators.

4. The balanced constraint is achieved using repair operators (optional).

5. The manufacturing constraints are implemented by adding penalty terms to the fitness function (optional).

6. Repeat steps 2-5 until one individual satisfies the stopping criterion.

The stacking sequence of each individual is represented by a string of genes in the chromosome, the values of the genes are $1,2,3$ and 4 , corresponding to ply orientations of $-45^{\circ}, 0^{\circ},+45^{\circ}$ and $90^{\circ}$, respectively. The size of the population in this paper is 50 with the roulette wheel method being employed in the selection procedure. The elitism operator is implemented to retain the best 2 individuals for the next generation without being changed. A two-point crossover operator is used with probability of $p_{c}=0.8$, the probability of mutation per gene is $p_{m}=0.1$. After mutation, permutation is applied to all chromosomes, randomly selecting two break points in the chromosome and reversing the order of the genes between these two points. The fitness function is formulated as

$$
\Gamma=\left[\sum_{i=1}^{3} \sum_{j=A}^{A, B, D} w_{j}\left|\xi_{i(\text { actual })}^{j}-\xi_{i(\text { target })}^{j}\right|\right]^{-1}+\alpha+\beta+\delta+\varepsilon
$$

where $\alpha, \beta, \delta$ and $\varepsilon$ are penalty terms for manufacturing constraints (1), (2), (3) and (4), above, respectively. A repair operator which is similar to that of Todoroki and Haftka [38] is employed in this paper to enforce the balanced constraint. The repair operator calculates the difference between the number of $+45^{\circ}$ plies and $-45^{\circ}$ plies for each individual. If the difference is even, half of the excess plies $\left(+45^{\circ}\right.$ or $\left.-45^{\circ}\right)$ are replaced by plies $\left(-45^{\circ}\right.$ or $+45^{\circ}$ ) from innermost excess plies. If the difference is odd, excess plies $\left(+45^{\circ}\right.$ or $\left.-45^{\circ}\right)$ are replaced by complementary plies $\left(-45^{\circ}\right.$ or $\left.+45^{\circ}\right)$ until there is only 1 excess ply left, and this ply is then replaced by one at $0^{\circ}$ or $90^{\circ}$. For the special situations when there is only one $+45^{\circ}$ or $-45^{\circ}$ ply and the number of complementary ply is 0 , the innermost $0^{\circ}$ or $90^{\circ}$ ply is replaced by the relative ply.

\section{Results and discussion}

Two sets of results are presented here. In the first set, the performance of the proposed two-level layup optimization is demonstrated with the efficiency of the logic-based method used in the second stage illustrated by comparison with a GA. In the second set, the target lamination parameters used in the logic-based method are taken directly 
from Refs. [39] to further demonstrate the capability of the technique for searching stacking sequences. All the results were obtained on a $4 \mathrm{GHz} \mathrm{PC}$.

\subsection{Layup optimization of a simply supported rectangular plate}

Firstly, layup optimization results are given for a simply supported rectangular plate loaded in longitudinal compression, the details of which are provided in Table 2. The required buckling load of this plate is $p_{d}=100 \mathrm{kN}$, and the lamination parameters relating to the original layup listed at the bottom of the table are $\xi_{1}^{A}=-0.1875$, $\xi_{2}^{A}=0.125, \xi_{3}^{A}=0.0625, \xi_{1}^{D}=-0.5251, \xi_{2}^{D}=0.2056, \xi_{3}^{D}=0.1643, \xi_{1,2,3}^{B}=0$. Different combinations of constraints are imposed on this plate.

Table 3 shows the optimized results from the first stage of the optimization taken from VICONOPT. After the first stage, the buckling loads $p_{c}$ of this plate under different constraints are all equal to the design load $p_{d}=100 \mathrm{kN}$, and the laminate thicknesses $h_{0}$ have reduced to around $3.5 \mathrm{~mm}$ allowing the number of plies $n$ to reduce from 32 to 28 . Therefore, it is clearly seen that $12.5 \%$ mass saving are achieved for these problems during the first stage. In the normal case which is not required to be symmetric or balanced, 9 lamination parameters are allowed to vary in the optimization, whereas in the symmetric case, $\xi_{1,2,3}^{B}=0$ are forced to be 0 , while $\xi_{3}^{A}$ is forced to be 0 when the plate is required to be balanced. The solution times of VICONOPT for these problems are all less than 1 second.

The lamination parameters listed in Table 3 are used as target values in the second stage optimization where layups under different design requirements are obtained using the logic-based method with all the weighting factors $w_{A, B, D}$ equal to 1 . There are six groups of layups in Table 4, which correspond to the six groups of required lamination parameters in Table 3.

It can be seen from the penultimate column of Table 4 that the global optimum results for all symmetric plates can be obtained within a very short time, usually followed by a longer time to complete the whole search. The exception is example 15 in which the global optimum result is obtained at 6.4 seconds, but a result with value of $\Gamma=0.1189$ which is very close to the global optimum result is obtained at 0.38 second.

Table 2

Properties and dimensions of the example plate.

\begin{tabular}{lc}
\hline$E_{1}\left(\mathrm{kN} / \mathrm{mm}^{2}\right)$ & 128 \\
$E_{2}\left(\mathrm{kN} / \mathrm{mm}^{2}\right)$ & 10.3 \\
$G_{1}\left(\mathrm{kN} / \mathrm{mm}^{2}\right)$ & 6.0 \\
$\rho\left(\mathrm{kg} / \mathrm{mm}^{3}\right)$ & 1.0 \\
$v_{12}$ & 0.3 \\
Length $(\mathrm{mm})$ & 150 \\
Width $(\mathrm{mm})$ & 100 \\
Ply thickness $\left(h_{p}\right)(\mathrm{mm})$ & 0.125 \\
Total thickness $\left(h_{0}\right)(\mathrm{mm})$ & 4.0 \\
Original layup & {$\left[90_{2} / 45 / 90 / 45 /-45 / 90_{2} / 45 /-45 / 0_{2} / 45 /-45 / 0 / 90\right] \mathrm{s}$}
\end{tabular}

Table 3

Stage 1 optimization results.

\begin{tabular}{|c|c|c|c|c|c|c|c|c|c|c|c|c|}
\hline Constraints & $\xi_{1}^{A}$ & $\xi_{2}^{A}$ & $\xi_{3}^{A}$ & $\xi_{1}^{D}$ & $\xi_{2}^{D}$ & $\xi_{3}^{D}$ & $\xi_{1}^{B}$ & $\xi_{2}^{B}$ & $\xi_{3}^{B}$ & $n$ & \multicolumn{2}{|c|}{$p_{c} / p_{d}$ time $(\mathrm{s})$} \\
\hline Normal & -0.168 & $-0.0854 c$ & 0.0097 & 0.0746 & -0.708 & $7-0.0261$ & -0.0072 & -0.0072 & -0.0072 & 28 & 1.0 & 0.785 \\
\hline 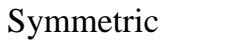 & $-0.1 \mathrm{C}$ & 06 & 0.03 & 0.02 & -0.7 & $2-0.03$ & 0 & 0 & 0 & 28 & 1.0 & 0.559 \\
\hline $0 \%$ & -0.088 & 0.255 & .0856 & 0.0628 & -0.8 & $3-0.0123$ & 0 & 0 & 0 & 28 & 1.0 & 0.502 \\
\hline ala & -0.1 & 0802 & 0 & 0.0299 & -0.8 & 7-0.0598 & -0.029 & -0.029 & -0.029 & 28 & 1.0 & 0.879 \\
\hline$y m+b a l$ & -0.15 & 0.0621 & 0 & 0.0437 & -0.79 & -0.0233 & 0 & 0 & 0 & 28 & 1.0 & 0.395 \\
\hline Sym+bal+10\% & -0.119 & -0.0585 & 0 & 0.0483 & -0.721 & $1-0.0196$ & 0 & 0 & 0 & 28 & 1.0 & 0.31 \\
\hline
\end{tabular}


Table 4

Stage 2 optimization results obtained from logic-based method.

\begin{tabular}{|c|c|c|c|c|c|c|c|}
\hline Example & Constraints & $\begin{array}{l}\text { Total } \\
\text { plies }\end{array}$ & Layup & $\Gamma$ & $p_{c} / p_{d}$ & $\begin{array}{l}\text { Time to } \\
\text { optimum } \\
\text { soln }(\mathrm{s})\end{array}$ & $\begin{array}{l}\text { Total } \\
\text { time } \\
(\mathrm{s})\end{array}$ \\
\hline 1 & Normal & 28 & $\begin{array}{l}{\left[-45_{2} / 45 /-45 / 45_{2} / 02 / 45 / 904 / 45 / 905 /(0 /\right.} \\
\left.-45)_{2} / 45 /-45_{2} / 45_{2}\right]\end{array}$ & 0.0806 & 1.0 & 4.77 & $3600+$ \\
\hline 2 & Symmetric & 28 & {$[45 /-45 / 45 /-45 /-45 / 45 /-45 / 02 / 905] \mathrm{s}$} & 0.1729 & 1.0 & 0.52 & 7.1 \\
\hline 3 & Sym+contiguity & 28 & {$\left[45 /-452 / 45 /-45 / 45 / 0 /-45 / 45 / 90_{2} / 0 / 90_{2}\right] \mathrm{s}$} & 0.1768 & 1.0 & 0.31 & 7.4 \\
\hline 4 & Sym+disorientation & 28 & {$\left[-45_{2} / 0 / 454 / 90 /-45 / 903 /-45 / 0\right] \mathrm{s}$} & 0.4705 & 0.97 & 0.12 & 0.95 \\
\hline 5 & Sym+disorientation & 29 & {$\left[-45_{2} / 0 / 454 / 90 /-45 / 903 /-45 / 0_{2}\right]_{\mathrm{MS}}$} & 0.4254 & 1.06 & 0.9 & 1.4 \\
\hline 6 & Sym $+10 \%$ & 28 & {$\left[45 /-45_{3} / 453 / 0_{2} /-45 / 90 / 45 / 90_{2}\right] \mathrm{s}$} & 0.0658 & 1.0 & 0.23 & 5.87 \\
\hline 7 & $\begin{array}{l}\text { Sym+10\%+contiguity } \\
+ \text { damtol }\end{array}$ & 28 & {$\left[45 /-453 / 453 / 02 /-45 / 90 / 45 / 90_{2}\right] \mathrm{s}$} & 0.0658 & 1.0 & 0.29 & 5.3 \\
\hline 8 & $\begin{array}{l}\text { Sym+10\%+contiguity } \\
+ \text { damtol +disorientation }\end{array}$ & 28 & {$[-452 / 0 / 454 / 90 / 45 / 90 /-45 / 90 /-45 / 0] \mathrm{s}$} & 0.3404 & 0.97 & 0.55 & 0.64 \\
\hline 9 & $\begin{array}{l}\text { Sym+10\%+contiguity } \\
\text { +damtol +disorientation }\end{array}$ & 29 & {$[-452 / 0 / 454 / 90 /-45 / 90 / 45 / 0 /-45 / 902]_{\mathrm{MS}}$} & 0.33 & 1.08 & 0.23 & 0.91 \\
\hline 10 & Balanced & 28 & $\begin{array}{l}{\left[-45 / 45 /(45 /-45)_{2} / 0 /-45 / 0 / 45 / 90_{7} / 02 / 90 /\right.} \\
\left.45_{2} /-45 / 45 /-453 / 45\right]\end{array}$ & 0.0892 & 1.0 & 3.76 & $3600+$ \\
\hline 11 & Sym+bal & 28 & {$[-45 / 45 /-45 / 452 /-45 / 0 / 45 /-45 / 90 / 0 / 903] \mathrm{s}$} & 0.0984 & 1.0 & 0.23 & 5.95 \\
\hline 12 & Sym+bal+contiguity & 28 & {$[-45 / 45 /-45 / 452 /-45 / 0 / 45 /-45 / 902 / 0 / 902] \mathrm{s}$} & 0.1115 & 1.0 & 0.29 & 6.34 \\
\hline 13 & Sym+bal+disorientation & 28 & {$[-452 / 0 / 454 / 90 /-45 / 903 /-45 / 0] \mathrm{s}$} & 0.3828 & 0.97 & 0.19 & 0.9 \\
\hline 14 & Sym+bal+disorientation & 29 & {$\left[-452 / 0 / 454 / 90 /-45 / 903 /-45 / 0_{2}\right]_{\mathrm{MS}}$} & 0.3776 & 1.07 & 0.97 & 1.59 \\
\hline 15 & Sym+bal+10\% & 28 & {$[45 /-452 / 452 /-45 / 0 /-45 / 0 / 904 / 45] \mathrm{s}$} & 0.112 & 1.0 & 6.4 & 9.06 \\
\hline 16 & $\begin{array}{l}\text { Sym+bal+10\% } \\
\text { +contiguity+damtol }\end{array}$ & 28 & {$\left[45 /-45_{2} / 45_{2} /-45 / 0 /-45 / 0 / 904 / 45\right] \mathrm{s}$} & 0.112 & 1.0 & 0.30 & 6.55 \\
\hline 17 & $\begin{array}{l}\text { Sym+bal+10\% } \\
\text { +contiguity+damtol } \\
\text { +disorientation }\end{array}$ & 28 & {$[-452 / 0 / 454 / 90 /-45 / 903 /-45 / 0] \mathrm{s}$} & 0.3307 & 0.97 & 0.49 & 1.01 \\
\hline 18 & $\begin{array}{l}\text { Sym+bal+10\% } \\
\text { +contiguity+damtol } \\
\text { +disorientation }\end{array}$ & 29 & {$\left[-45_{2} / 0 / 454 / 90 /-45 / 903 /-45 / 0_{2}\right]_{\mathrm{MS}}$} & 0.2853 & 1.07 & 0.69 & 1.37 \\
\hline
\end{tabular}

As the problem size increases, the total searching time will be longer, for example, as the number of layers of the normal case is double of these symmetric cases, around $4 \times 10^{28}$ new possibilities are added to the normal case. A promising result is obtained at 4.77 seconds but it cannot be guaranteed as the global optimum result until the whole search is completed which takes more than 1 hour. Therefore, the search is forced to stop after the 9 th cycle in this paper (around 90 seconds), because a very low value of $\Gamma$ can be obtained early in the first few cycles, and the time for completing the decision tree search starts increasing dramatically at this point, being around 9 seconds, 80 seconds and 1200 seconds in the 8th, 9th and 10th cycles, respectively. Nevertheless, for practical design, the search can be stopped as soon as an acceptable result is found.

The contiguity, disorientation and damtol constraints are only introduced in the second stage. Owing to the nature of thecontiguity and damtol constraints, they only make minimal changes to the stacking sequence, and hence have only a small impact on the buckling load.

Fig. 7 shows the difference between the actual lamination parameters and the target values corresponding to the 18 examples listed in Table 4. It is clearly seen that the differences are quite small as values of $\Gamma$ are around 0.1 except when adding the disorientation constraint which cause a bad mismatch on $\xi_{2}^{D}$. Fig. 8 compares the actual lamination parameters of examples 15,16 and 17 against the target lamination parameters in the lamination parameters space. Target lamination parameters are represented by a blue circle. As examples 15 and 16 have the same actual lamination parameters they are both represented by red crosses, while the actual lamination parameters of example 17 are represented by black crosses. It can be seen that these three examples have same $\xi_{1,2,3}^{A}$, which means that the three examples under different manufacturing constraints have same number of plies for each angle, hence the difference between these cases are caused only by the stacking sequences. The distances between the actual lamination parameters and target values for the $\xi_{1,2,3}^{A}$ are small. As can be seen from Figs. 8 (c) and (d), the 
contiguity and damtol constraints have no effect on the stacking sequence in example 16 and very good matches are achieved for the out-of-plane lamination parameters. However, the disorientation constraint which introduces more limitations in choosing the stacking sequences makes the $\xi_{2}^{D}$ far from the target in example 17.

Because the disorientation constraint significantly reduces the search space, the total searching time for examples under the disorientation constraint is around 1 second which is much shorter than the other examples as shown in Table 4. However the presence of the disorientation constraint makes it quite easy to violate the buckling constraint. The buckling loads $p_{c}$ for the examples which involve the use of the disorientation constraint are slightly lower than the design load $p_{d}$, and so these examples were re-run with the number of plies increased to 29 , which increased $p_{c}$ by around $10 \%$ and thus satisfied the buckling constraint. Note that some examples in Table 4 have identical optimal layups but different values of $\Gamma$ due to their different target values of lamination parameters.

As shown in Table 5, four symmetric examples listed in Table 4 were re-run using GAs in order to make a direct comparison with the logic-based method in optimizing stacking sequences. Since GAs search randomly for each run, each GA was run 10 times for each example to guarantee the reliability of these comparisons. The times for

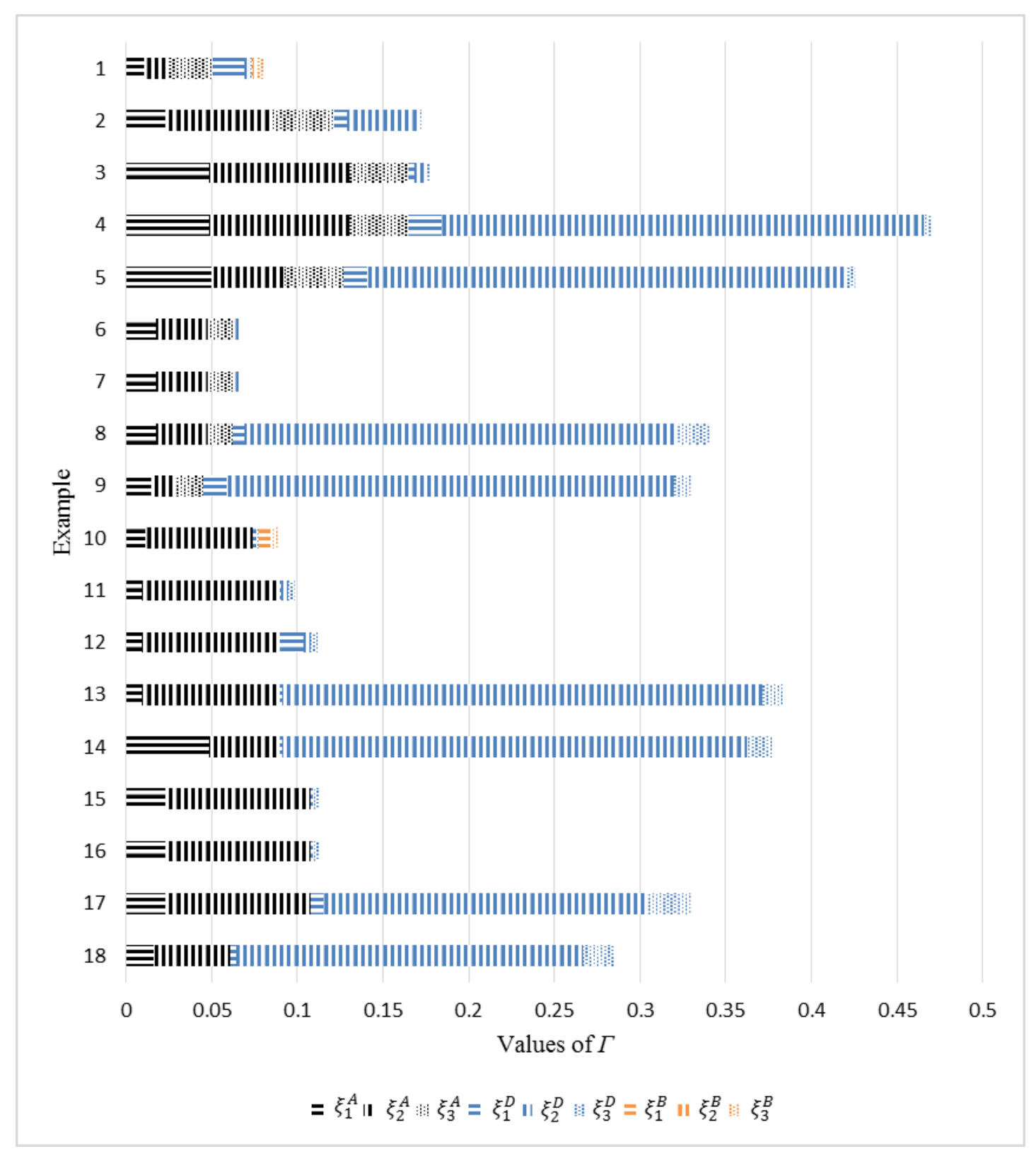

Fig. 7. The difference between the target lamination parameters and actual lamination parameters for the 18 cases. 


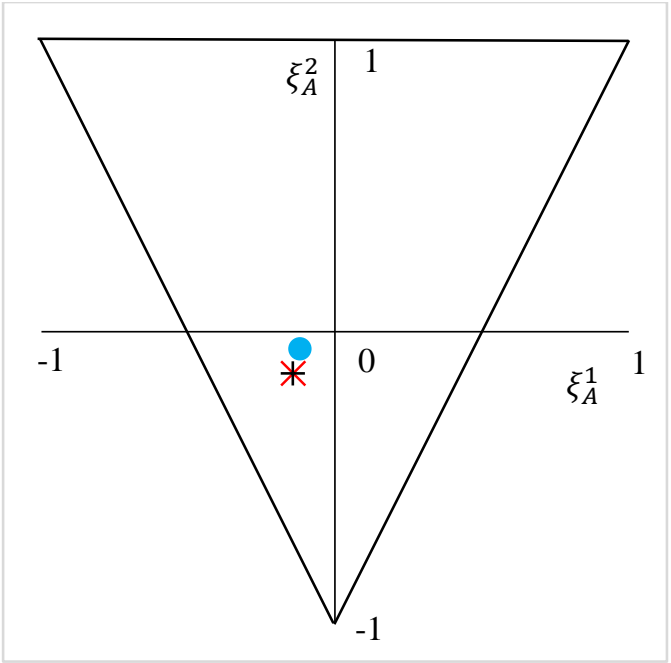

(a)

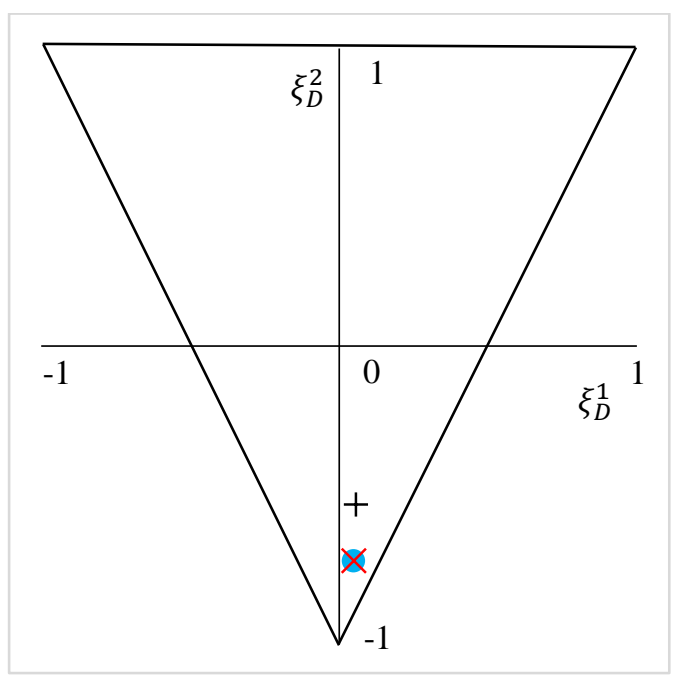

(c)

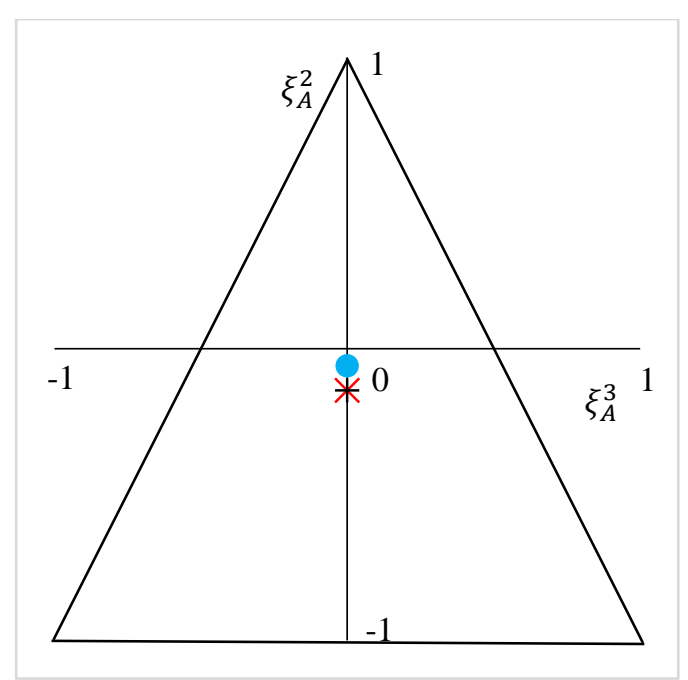

(b)

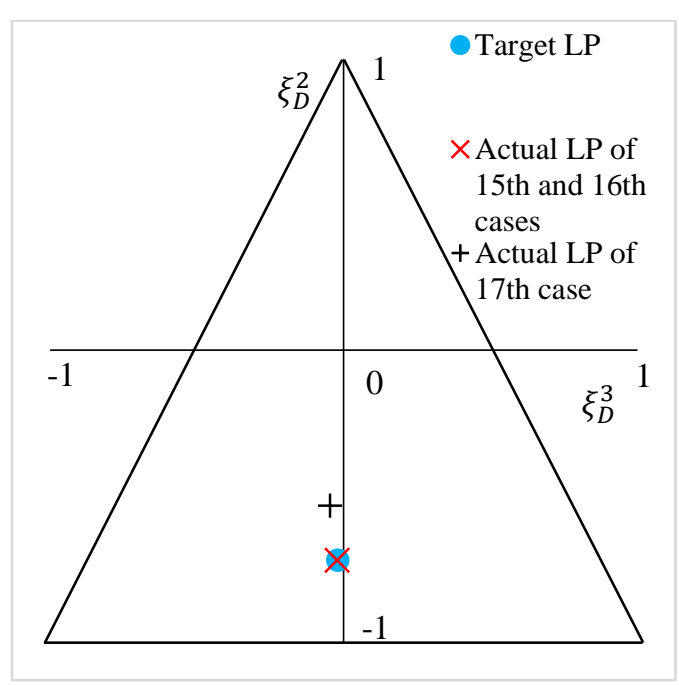

(d)

Fig. 8. Plot of actual lamination parameters against the target lamination parameters on lamination parameters space.

obtaining the optimal result for each run are listed from the best to the worst in Table 5. It is observed that the GA almost always takes longer to find the global optimal result and is only faster than the logic-based method in one case. In the last column of Table 5, as discussed above, the disorientation constraint makes the total searching time shorter for the logic-based method as more branches can be discarded logically. However, it causes longer solution times for GAs, because the disorientation constraint can be easily violated by the crossover and mutation operators of GAs during its stochastic search. A comparison between the solution times for the logic-based method and the best 5 GAs for the symmetric case is shown in Fig. 9 (a), where the blue circle represents the global optimal result obtained from the logic-based method and the triangle in other colors represent the global optimal results given by the GAs. It can be seen that the best GA run (shown in red) obtains the optimal result at 0.37 second which is faster than the logic-based method, but before this time point, the logic-based method found intermediate solutions quicker than all the GAs. Note that the end of the blue line indicates the end of the logic-based search during which all the possible layups have been logically searched, confirming that the global optimal result has been obtained. However, although all the GAs eventually found the same result, they could not confirm its optimality. Table 5 shows that the remaining 5 GAs (not shown in Fig. 9 (a)) took a very long time to find the global optimal result. This is because these runs obtained a layup $[45 /-45 /(-45 / 45) 2 / 0 /-45 / 45 / 902 / 0 / 902] \mathrm{s}$ with $\Gamma=0.17684$ or a layup [($\left.45 / 45)_{3} / 45 / 0 / 90 /-45 / 0 / 903\right] s$ with $\Gamma=0.1784$ at an early stage. These $\Gamma$ are close to the optimal value, but the corresponding layups are far away from the optimum layup, and it subsequently took a long time for the GAs to move away from these local optimum results. 
Table 5

Comparison between GAs and logic-based method for four symmetric examples from Table 4.

\begin{tabular}{lcccc}
\hline & $\begin{array}{c}\text { Example 2 } \\
\text { Symmetric } \\
(\Gamma=0.1729)\end{array}$ & $\begin{array}{c}\text { Example 11 } \\
\text { Sym+bal } \\
(\Gamma=0.0984)\end{array}$ & $\begin{array}{c}\text { Example 16 } \\
\text { Sym+bal+contiguity } \\
+ \text { min\%+damtol } \\
(\Gamma=0.112)\end{array}$ & $\begin{array}{c}\text { Example 18 } \\
\text { Sym+bal+contiguity+min\% } \\
\text { +damtol+disorientation } \\
(\Gamma=0.2853)\end{array}$ \\
\hline GAs & 0.37 & 0.29 & 0.47 & 0.91 \\
Time for obtaining the & 0.71 & 0.62 & 0.88 & 0.95 \\
optimal result (s) & 2.28 & 1.29 & 1.46 & 1.38 \\
& 9.17 & 1.32 & 1.89 & 5.57 \\
& 14.69 & 1.39 & 2.73 & 6.15 \\
& 39.02 & 1.40 & 2.75 & 11.43 \\
& 47.28 & 3.14 & 4.57 & 16.03 \\
& 56.86 & 5.79 & 6.03 & 17.40 \\
& 89.59 & 8.02 & 9.77 & 42.74 \\
\cline { 2 - 5 } & 136.73 & 9.79 & 17.80 & 0.69 \\
Time for obtaining the & 0.52 & & & \\
optimal result (s) & & 0.23 & 0.3 & 1.37 \\
Total solution time (s) & 7.1 & 5.95 & 6.55 & \\
\hline
\end{tabular}

Table 6

Comparison between GAs and logic-based method for two non-symmetric examples from Table 4.

\begin{tabular}{lcccc}
\hline & $\begin{array}{c}\text { Example 1 } \\
\text { Normal }\end{array}$ & $\begin{array}{c}\text { Time for obtaining } \\
\text { this result (s) }\end{array}$ & $\begin{array}{c}\text { Example 10 } \\
\text { Balanced }\end{array}$ & $\begin{array}{c}\text { Time for obtaining } \\
\text { this result (s) }\end{array}$ \\
\hline GAs & & & & \\
Values of $\Gamma$ after & 0.0851 & 33.76 & 0.0911 & 133.20 \\
300 seconds & 0.0938 & 219.98 & 0.0926 & 55.11 \\
& 0.0956 & 88.10 & 0.0926 & 119.39 \\
& 0.1000 & 16.97 & 0.0932 & 42.20 \\
& 0.1087 & 12.56 & 0.0945 & 191.41 \\
& 0.1101 & 108.57 & 0.0965 & 4.25 \\
& 0.1113 & 3.70 & 0.0965 & 277.41 \\
& 0.1113 & 9.62 & 0.0967 & 8.39 \\
Logic-based method & 0.1135 & 99.67 & 0.0967 & 50.09 \\
Values of $\Gamma$ after & 0.1235 & 246.16 & 0.0977 & 7.11 \\
300 seconds & 0.0806 & 4.77 & & \\
\hline
\end{tabular}

To investigate the efficiency of the logic-based method with larger number of plies in comparison with GAs, the normal and balanced cases of which the real number of plies in the decision trees are double of those of symmetric cases were re-run 10 times using GAs. Since GAs cannot find the same results as the logic-based method for a long time, GAs and the logic-based method were both run for 300 seconds and the comparisons were made for the final values of $\Gamma$ which are listed from the best to the worst in Table 6. For both cases, the logic-based method can find very good results efficiently, however, GAs cannot achieve the same values of $\Gamma$ as the logic-based method even after 300 seconds. It is observed that the advantage of the logic-based method is more obvious with larger numbers of plies, because it is easier to match the target lamination parameters. However, a larger number of plies also leads to more local optimum results in the second stage optimization, and more plies need to be changed to move away from these local optimum results, which is to the disadvantage of the stochastic search of GAs. A comparison between the solution times for the logic-based method and the best 5 GAs for the normal case is shown in Fig. 9 (b), where the blue circle represents the final result obtained by the logic-based method in 300 seconds and the triangles in other colors represent the final results given by the GAs. It can be seen that the logic-based method always takes the lead in the comparison over 300 seconds. These comparisons confirm the advantages of the proposed logic-based search over stochastic-based methods in quickly obtaining reliable results, making it 
more appropriate for layup optimization. Note that due to the manufacturing requirement, the ply angles are usually restricted to $0^{\circ}, 90^{\circ},+45^{\circ}$ and $-45^{\circ}$, and only these four angles have been considered in this paper.

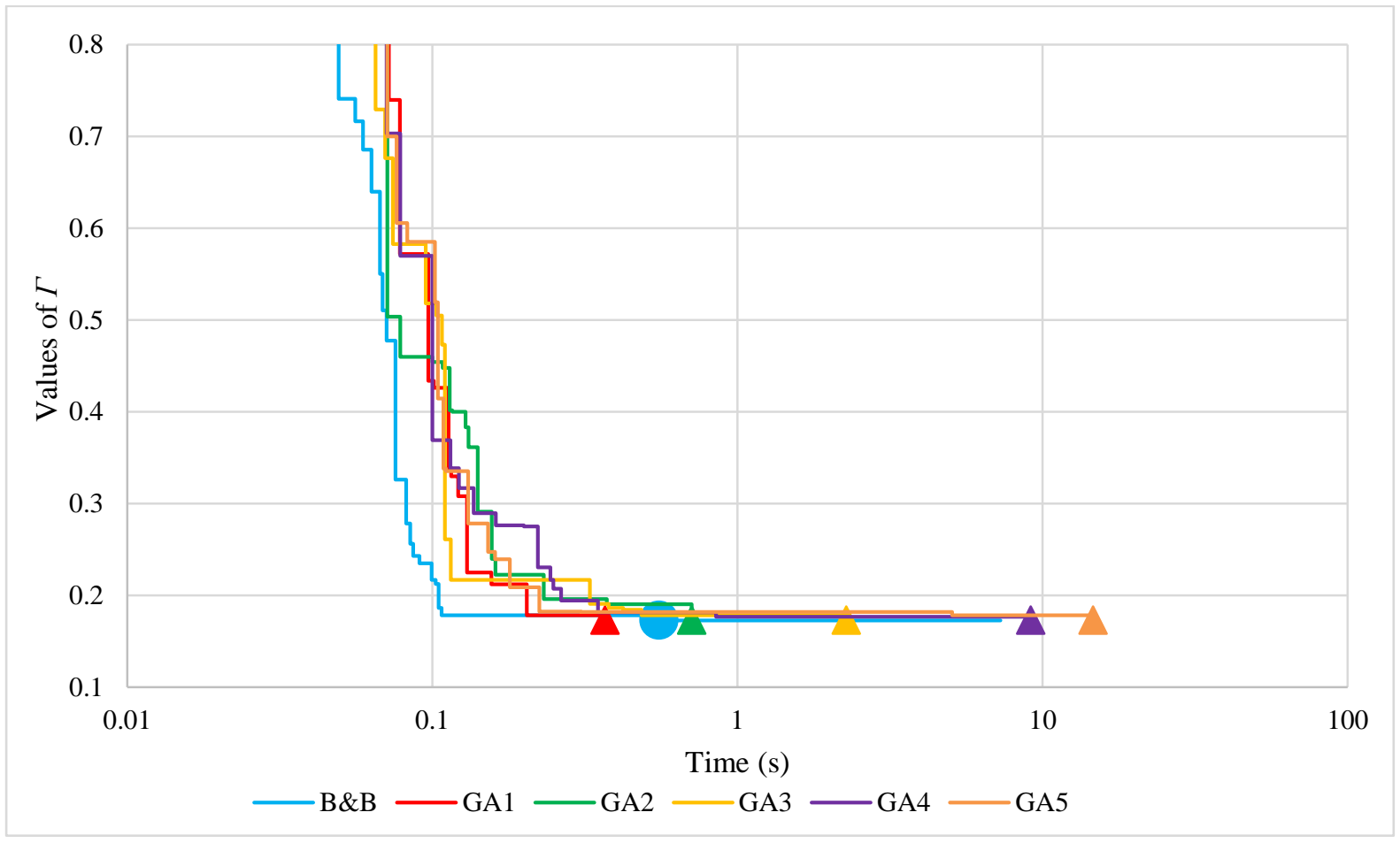

(a)

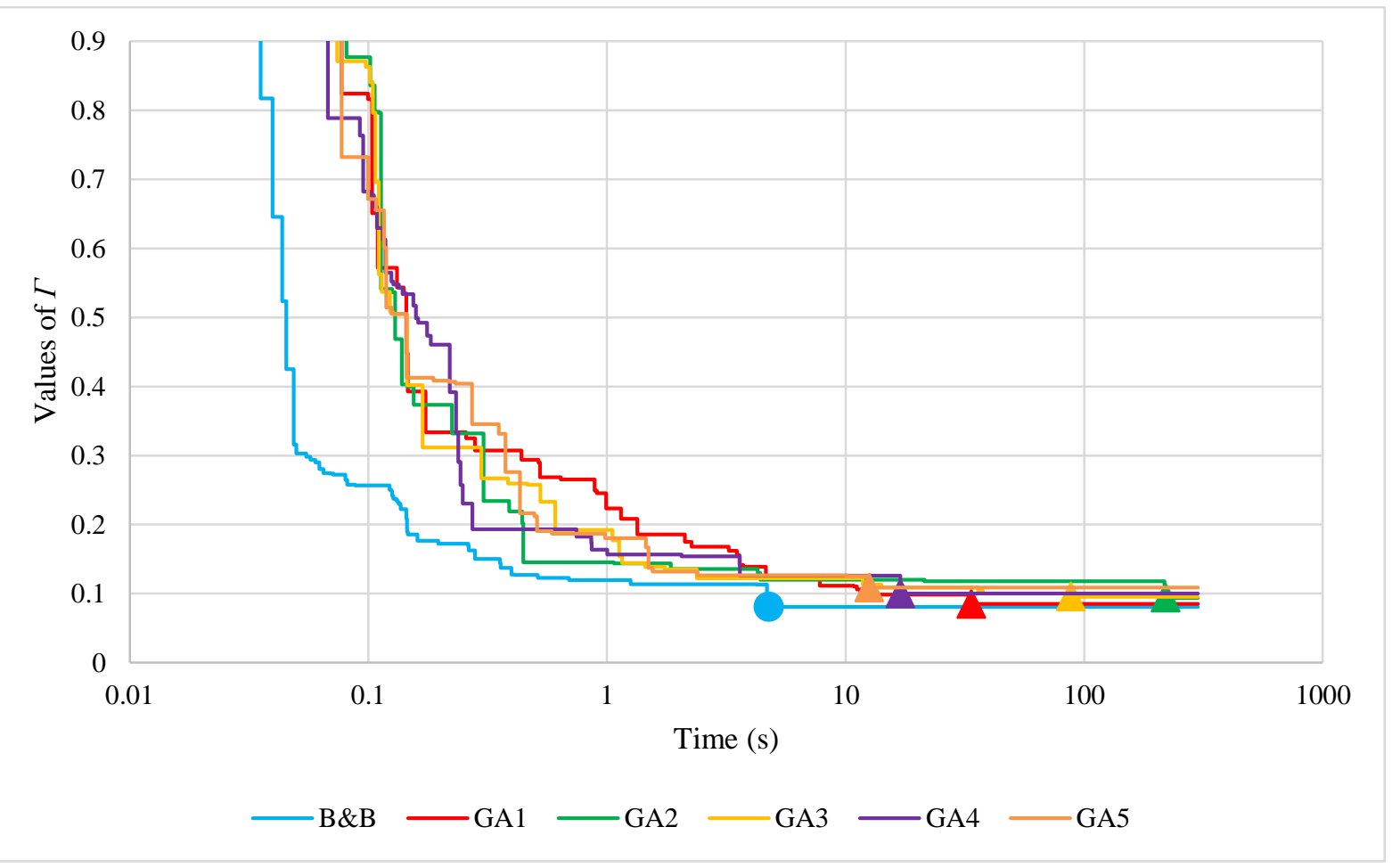

(b)

Fig. 9. Comparison between GAs and logic-based method. (a) Example 2: Symmetric. (b) Example 1: Normal. 


\subsection{Layup optimization of stiffened panels}

Secondly, to demonstrate the capability of the logic-based method for searching layups, target lamination parameters for a range of stiffened panels are taken directly from Refs. [39] with the same number of plies being assumed for comparison purpose. Here the comparison is focused on which method can find stacking sequences which are closer to the target lamination parameters and also satisfy the required constraints. Ref. [39] employed GAs in which the fitness function was built by calculating the squared differences between the optimum and actual lamination parameters.

The optimal lamination parameters are shown in Table 7, while the optimized stacking sequences for each example are listed in Table 8. The constraints used for each laminate for the logic-based method are the same as those in the second stage in Refs. [39] (i.e. contiguity constraint, minimum percentage constraint and a special case of damtol constraint requiring at least one set of \pm 45 plies at the skin and stiffener surfaces). The stacking sequence in Ref. [39] for the skin of example C slightly violates the $10 \%$ constraint but within an acceptable tolerance, while the result given by the logic-based method satisfies this constraint because these constraints are enforced during the logic-based search. It can be seen from Table 7, that for examples A, B and D, the $\xi_{1,2,3}^{A}$ of the laminates in this paper are same as those in Ref. [39], meaning that they have same strength, percentage of each ply orientation and Poisson's ratio mismatch. However the results of this paper have a better match on $\xi_{1,2,3}^{D}$ which determine the buckling performance of each laminate, and hence lower values of $\Gamma$ are obtained by logic-based method. As expected, the buckling load factors for these three optimized configurations obtained in Ref. [39] are 1.117, 1.09 and 0.989 , while those given by the logic-based method have higher values of 1.124, 1.097 and 1.029, respectively, hence with an improvement of buckling performance between $0.6 \%$ and $4.0 \%$. Note that, to make a fair comparison, the buckling load factors presented in this paper are all calculated by VICONOPT. Therefore, for the optimized configurations obtained in Ref. [39], their buckling load factors presented herein are slightly different

Table 7

Optimum lamination parameters and $\Gamma$ for three methods.

\begin{tabular}{|c|c|c|c|c|c|c|c|c|}
\hline Example & Method & $\xi_{1}^{A}$ & $\xi_{2}^{A}$ & $\xi_{3}^{A}$ & $\xi_{1}^{D}$ & $\xi_{2}^{D}$ & $\xi_{3}^{D}$ & $\Gamma$ \\
\hline A & first stage & 0.4603 & 0.3206 & 0.1208 & -0.0028 & -0.2908 & 0.3911 & \\
\hline Skin & second stage (Ref.39) & 0.4237 & 0.2542 & 0.1695 & 0.1346 & -0.2123 & 0.2672 & 0.4915 \\
\hline (59 plies) & second stage (this paper) & 0.4237 & 0.2542 & 0.1695 & 0.1315 & -0.2719 & 0.3998 & 0.3136 \\
\hline A & first stage & 0.5862 & 0.5724 & 0.0002 & 0.1603 & -0.0281 & 0.0016 & \\
\hline Stiffener & second stage (Ref.39) & 0.4839 & 0.4839 & 0 & 0.2261 & 0.0102 & -0.0532 & 0.3499 \\
\hline (31 plies) & second stage (this paper) & 0.4839 & 0.4839 & 0 & 0.2261 & 0.0102 & -0.0532 & 0.3499 \\
\hline $\mathrm{B}$ & first stage & 0.4551 & 0.3102 & 0.1449 & 0.0434 & -0.1356 & 0.2124 & \\
\hline Skin & second stage (Ref.39) & 0.4483 & 0.3103 & 0.1379 & 0.1098 & -0.0872 & 0.2314 & 0.1477 \\
\hline (58 plies) & second stage (this paper) & 0.4483 & 0.3103 & 0.1379 & 0.095 & -0.1364 & 0.2078 & 0.071 \\
\hline B & first stage & 0.5934 & 0.5868 & 0.0009 & 0.1674 & -0.0011 & 0.0036 & \\
\hline Stiffener & second stage (Ref.39) & 0.4894 & 0.4894 & 0 & 0.2744 & 0.0862 & -0.0079 & 0.4081 \\
\hline (47 plies) & second stage (this paper) & 0.4894 & 0.4894 & 0 & 0.2698 & -0.0035 & -0.0055 & 0.3162 \\
\hline $\mathrm{C}$ & first stage & 0.4850 & 0.3700 & 0.0866 & -0.0185 & -0.1623 & 0.2797 & \\
\hline Skin & second stage (Ref.39) & 0.4426 & 0.2787 & 0.0984 & 0.1303 & -0.1244 & 0.1853 & 0.4266 \\
\hline (61 plies) & second stage (this paper) & 0.4426 & 0.3443 & 0.0656 & 0.2572 & -0.1643 & 0.2829 & 0.3703 \\
\hline $\mathrm{C}$ & first stage & 0.3941 & 0.1881 & 0 & 0.3239 & -0.2744 & 0.103 & \\
\hline Flange & second stage (Ref.39) & 0.3333 & 0.1111 & 0 & 0.3416 & -0.2785 & 0.1235 & 0.1799 \\
\hline (18 plies) & second stage (this paper) & 0.3333 & 0.1111 & 0 & 0.3416 & -0.2785 & 0.1235 & 0.1799 \\
\hline $\mathrm{C}$ & first stage & 0.7541 & 0.9083 & -0.0198 & 0.6044 & 0.7372 & -0.0542 & \\
\hline Web & second stage (Ref.39) & 0.625 & 0.75 & 0 & 0.6016 & 0.7559 & -0.0396 & 0.3433 \\
\hline (32 plies) & second stage (this paper) & 0.5625 & 0.875 & 0.0625 & 0.6013 & 0.9966 & 0.0017 & 0.6256 \\
\hline $\mathrm{D}$ & first stage & 0.4887 & 0.3775 & 0.0796 & 0.0217 & -0.2271 & 0.2897 & \\
\hline Skin & second stage (Ref.39) & 0.4444 & 0.3968 & 0.0794 & 0.2312 & -0.0932 & 0.1409 & 0.5560 \\
\hline (63 plies) & second stage (this paper) & 0.4444 & 0.3968 & 0.0794 & 0.1792 & -0.1888 & 0.2824 & 0.2669 \\
\hline $\mathrm{D}$ & first stage & -0.0277 & -0.3359 & 0 & 0.2961 & -0.0299 & 0.0473 & \\
\hline Flange & second stage (Ref.39) & 0 & 0 & 0 & -0.0937 & -0.75 & 0.2813 & 1.7075 \\
\hline (8 plies) & second stage (this paper) & 0 & 0 & 0 & -0.0937 & -0.75 & 0.2813 & 1.7075 \\
\hline $\mathrm{D}$ & first stage & 0.6947 & 0.7412 & 0 & 0.6671 & 0.337 & 0.0321 & \\
\hline Web & second stage (Ref.39) & 0.6226 & 0.6981 & 0 & 0.7464 & 0.5427 & -0.0181 & 0.4504 \\
\hline (53 plies) & second stage (this paper) & 0.6226 & 0.6981 & 0 & 0.6268 & 0.3557 & 0.038 & 0.1801 \\
\hline
\end{tabular}


Table 8

Optimum stacking sequences for three methods.

\begin{tabular}{|c|c|c|c|c|c|}
\hline \multirow[t]{2}{*}{ Example } & \multirow[t]{2}{*}{ Layup } & \multicolumn{4}{|c|}{ Percentage for each angle $(\%)$} \\
\hline & & $0^{\circ}$ & $90^{\circ}$ & $+45^{\circ}$ & $-45^{\circ}$ \\
\hline $\bar{A}$ & {$\left[ \pm 45 / 45_{3} / 90_{2} /\left( \pm 45 / 0_{4}\right)_{2} / 45 / 0_{4} / 45 / 0_{2} / 90 / 0 / 0\right]_{\mathrm{MS}}$} & 52.54 & 10.17 & 27.12 & 10.17 \\
\hline Skin & this paper $\left[ \pm 45 / 454 /(90 / 45)_{2} / 0_{4} /-45 / 0_{4} / 45 / 0_{4} / 90 / 0_{3} /-45 / 0\right]_{\mathrm{MS}}$ & 52.54 & 10.17 & 27.12 & 10.17 \\
\hline A & {$\left[ \pm 45 / 90 /-45 / 0_{4} / 45 / 0_{4} / 90 / 0 / 0\right]_{\mathrm{MS}}$} & 61.29 & 12.9 & 12.9 & 12.9 \\
\hline Stiffener & this paper $\left[ \pm 45 / 90 /-45 / 0_{4} / 45 / 04 / 90 / 0 / 0\right]_{\mathrm{MS}}$ & 61.29 & 12.9 & 12.9 & 12.9 \\
\hline$\overline{\mathrm{B}}$ & {$\left[ \pm 45 / 90_{2} / 45 / \pm 45 / 0_{2} / 45_{2} /\left(45 / 0_{4}\right)_{2} / 90 / 04 /-45 / 0_{2}\right] \mathrm{s}$} & 55.17 & 10.34 & 24.14 & 10.34 \\
\hline Skin & this paper $\left[ \pm 45 /-45 / 45 / 90 / 45_{2} / 90 / 45 / 02 / 90 /(04 / 45)_{2} / 0_{4} /-45 / 0_{2}\right] \mathrm{s}$ & 55.17 & 10.34 & 24.14 & 10.34 \\
\hline $\mathrm{B}$ & Ref.39 $\left[( \pm 45)_{2} / 0_{2} / 90_{2} / 0_{4} /-45 / 04 / 90 / 0_{4} / 45 / 0\right]_{\mathrm{MS}}$ & 61.7 & 12.77 & 12.77 & 12.77 \\
\hline Stiffener & this paper $\left[ \pm 45 / 90 / 45 / 0 /-45_{2} / 0_{4} / 45 / 0_{4} / 90 / 0_{4} / 90 / 0_{2}\right] \mathrm{MS}$ & 61.7 & 12.77 & 12.77 & 12.77 \\
\hline $\mathrm{C}$ & {$\left[ \pm 45 / 45 / 902 / 45 /\left( \pm 45 / 0_{2}\right)_{2} / 0_{2} / 45 / 0_{3} / 45 / 0_{2} / 90 / 0_{4} / 45 / 0 / 0\right]_{\mathrm{MS}}$} & 54.1 & 9.84 & 26.23 & 9.84 \\
\hline Skin & this paper $\left[ \pm 45 / 45 / 90 / 454 / 0_{4} /-45 / 0_{4}\left(-45 / 0_{3}\right)_{2} / 90 / 0_{2} / 90 / 0 / 90\right]_{\mathrm{MS}}$ & 55.74 & 11.48 & 19.67 & 13.11 \\
\hline $\mathrm{C}$ & {$[ \pm 45 / 02 / 45 / 0 /-45 / 90 / 0] \mathrm{s}$} & 44.44 & 11.11 & 22.22 & 22.22 \\
\hline Flange & this paper $\left[ \pm 45 / 0_{2} / 45 / 0 /-45 / 90 / 0\right] \mathrm{s}$ & 44.44 & 11.11 & 22.22 & 22.22 \\
\hline $\mathrm{C}$ & Ref.39 $\quad\left[0_{2} / 90 / 0_{2} /-45 / 0_{2} / 45 / 0_{4} / 90 / 0_{2}\right] \mathrm{s}$ & 75 & 12.5 & 6.25 & 6.25 \\
\hline Web & this paper $\left[\left(0_{3} / 90\right)_{2} / 0 / 90 / 0_{4} / 45 / 0\right]_{\mathrm{s}}$ & 75 & 18.75 & 6.25 & 0 \\
\hline $\mathrm{D}$ & {$\left[ \pm 45 / 45 / \pm 45 / 0_{2} / 90 / \pm 45 / 45 / 0_{4} / 90 /\left(90 / 0_{4}\right)_{2} / 45 / 0_{4} /-45\right]_{\mathrm{MS}}$} & 57.14 & 12.7 & 19.05 & 12.7 \\
\hline Skin & this paper $\left[ \pm 45 / 454 /-45 / 45 / 90 / 0_{2} /\left(90 / 0_{4}\right)_{2} /-45 / 0_{4} / 90 / 0_{4} /-45\right] \mathrm{MS}$ & 57.14 & 12.7 & 19.05 & 12.7 \\
\hline $\mathrm{D}$ & {$[ \pm 45 / 90 / 0] \mathrm{s}$} & 25 & 25 & 25 & 25 \\
\hline Flange & this paper $[ \pm 45 / 90 / 0] \mathrm{s}$ & 25 & 25 & 25 & 25 \\
\hline $\mathrm{D}$ & {$\left[04 / \pm 45 / 0_{2} /-45 / 0_{4} / 45 /(04 / 90)_{2} / 90 / 0 / 0\right]_{\mathrm{MS}}$} & 73.58 & 11.32 & 7.55 & 7.55 \\
\hline Web & this paper $\left[45 /-45 / 0_{3} / 45 / 0_{4} /-45 /(04 / 90)_{3} / 0\right]_{\mathrm{MS}}$ & 73.58 & 11.32 & 7.55 & 7.55 \\
\hline
\end{tabular}

Note that the C skin in Ref [39] slightly violates the $10 \%$ constraint. The reason why the C and D webs shown in this table appear to violate the $10 \%$ constraint is because those are actually plates which are sandwiched by flanges on both sides to constitute the actual web, as described in Refs [39].

from those values listed in Ref. [39]. The results suggest that the logic-based method has better capability in searching the stacking sequences, this is because sometimes GAs have the weakness of missing the global optimum or can take too long time to find it for problems with many plies, whereas useful results can be obtained quite quickly by the logic-based method.

\section{Conclusions}

An efficient two-stage method to perform global layup optimization of composite laminates under compression, subject to buckling, manufacturing and lamination parameter constraints, has been presented in this paper. VICONOPT is employed in the first stage to conduct a gradient-based optimization process using lamination parameters. The optimized lamination parameters are treated as target values when searching corresponding layups in the second stage where a logic-based method is employed. The logic-based method is a combination of the branch and bound method and a global layerwise technique, which can find optimum results for different combinations of constraints (e.g. symmetric, balanced and manufacturing constraints). Restricted feasible regions for the lamination parameters for a $10 \%$ constraint is studied in this paper, showing that the feasible regions for $\xi_{1,2,3}^{A}$ are reduced by $64 \%$, while the feasible regions for $\xi_{1,2,3}^{D}$ is only reduced by around $3 \%$. In the first set of results, $12.5 \%$ mass savings are achieved efficiently for a plate subjected to compression load under different constraints using this two-stage optimization, and the efficiency of the logic-based method is illustrated by comparison with a GA, showing that the logic-based method is almost always faster in layup optimization. In the second set of results, the logic-based method is compared with previously published results by taking their target lamination parameters and numbers of plies. The results confirm that the logic-based method can find results closer to the target values, and hence give a slightly better buckling performance (with an improvement of $0.6 \%$ to $4.0 \%$ ).

\section{Acknowledgement}

The first author would like to thank China Scholarship Council (CSC) for the financial support. 


\section{References}

[1] Jones RM. Mechanics of Composite Materials. Taylor and Francis; 1999

[2] Fielding JP. Introduction to aircraft design. Cambridge University Press; 1999.

[3] Todoroki A, Ishikawa T. Design of experiments for stacking sequence optimizations with genetic algorithm using response surface approximation. Compos Struct 2004;64:349-57. doi:10.1016/j.compstruct.2003.09.004.

[4] Hajmohammad MH, Salari M, Hashemi SA, Esfe MH. Optimization of stacking sequence of composite laminates for optimizing buckling load by neural network and genetic algorithm. Indian J Sci Technol 2013;6:5070-7. doi:10.17485/IJST/2013/V6I8/36346.

[5] Wittrick WH, Williams FW. Buckling and vibration of anisotropic or isotropic plate assemblies under combined loadings. Int J Mech Sci 1974;16:209-39. doi:10.1016/0020-7403(74)90069-1.

[6] M.S.Anderson., F.W.Williams., C.J.Wright. Buckling and vibration of any prismatic assembly of shear and compression loaded anisotropic plates with an arbitrary supporting structure. Int J Mech Sci 1983;25:585-96. doi:10.1016/0020-7403(83)90050-4.

[7] Kennedy D, Featherston CA. Exact strip analysis and optimum design of aerospace structures. Aeronaut J 2010;114:505-12.

[8] Butler R, Williams FW. Optimum design using viconopt, a buckling and strength constraint program for prismatic assemblies of anisotropic plates. Comput Struct 1992;43:699-708. doi:10.1016/00457949(92)90511-W.

[9] Liu W, Butler R. Buckling optimization for composite panels with elastic tailoring. 49th AIAA/ASME/ASCE/AHS/ASC Struct Struct Dyn Mater Conf 2008:1-10.

[10] Liu W, Butler R. Optimum Buckling Design of Composite Wing Cover Panels. Optimization 2007:111.

[11] Le Riche R, Haftka RT. Optimization of laminated stacking sequence for buckling load maximization by genetic algorithm. Aiaa 1992;31:951-6. doi:10.2514/3.11710.

[12] Park JH, Hwang JH, Lee CS, Hwang W. Stacking sequence design of composite laminates for maximum strength using genetic algorithms. Compos Struct 2001;52:217-31. doi:10.1016/S02638223(00)00170-7.

[13] Almeida FS, Awruch AM. Design optimization of composite laminated structures using genetic algorithms and finite element analysis. Compos Struct 2009;88:443-54. doi:10.1016/j.compstruct.2008.05.004.

[14] Chen S, Lin Z, An H, Huang H, Kong C. Stacking sequence optimization with genetic algorithm using a two-level approximation. Struct Multidiscip Optim 2013;48:795-805. doi:10.1007/s00158-013-0927-4.

[15] Miki M, Sugiyama Y. Optimum design of laminated composites plates using lamination parameters. AIAA J 1993;31:921-2. doi:10.2514/3.49033.

[16] Yamazaki K. Two-level optimization technique of composite laminate panels by genetic algorithms. Collect Tech Pap - AIAA/ASME/ASCE/AHS/ASC Struct Struct Dyn Mater Conf 1996;3:1882-7. doi:doi:10.2514/6.1996-1539.

[17] Diaconu CG, Sato M, Sekine H. Feasible region in general design space of lamination parameters for laminated composites. AIAA J 2002;40:559-65. doi:10.2514/3.15097.

[18] Diaconu CG, Sekine H. Layup optimization for buckling of laminated composite shells with restricted layer angles. Aiaa J 2004;42:2153-63. doi:10.2514/1.931.

[19] Bloomfield M., Diaconu C., Weaver P. On feasible regions of lamination parameters for lay-up optimization of laminated composites. Proc R Soc A Math Phys Eng Sci 2009;465:1123-43. 
doi:10.1098/rspa.2008.0380.

[20] Wu Z, Raju G, Weaver PM. Feasible Region of Lamination Parameters for optimization of Variable Angle Tow (VAT) Composite Plates. 54th AIAA/ASME/ASCE/AHS/ASC Struct Struct Dyn Mater Conf 2013:1-10. doi:10.2514/6.2013-1481.

[21] Gubarev F, Kunin V, Pospelov a. Lay-up Optimization of Laminated Composites: Mixed Approach with Exact Feasibility Bounds on Lamination Parameters 2013:1-5.

[22] Herencia JE, Weaver PM, Friswell MI. Optimization of anisotropic composite panels with T-shaped stiffeners including transverse shear effects and out-of-plane loading. Struct Multidiscip Optim 2008;37:165-84. doi:10.1007/s00158-008-0227-6.

[23] Herencia JE, Haftka RT, Weaver PM, Friswell MI. Lay-Up Optimization of Composite Stiffened Panels Using Linear Approximations in Lamination Space. AIAA J 2008;46:2387-91. doi:10.2514/1.36189.

[24] Wu Z, Raju G, Weaver PM. Framework for the Buckling Optimization of Variable-Angle Tow Composite Plates. AIAA J 2015;53:3788-804. doi:10.2514/1.J054029.

[25] Bloomfield MW, Herencia JE, Weaver PM. Enhanced two-level optimization of anisotropic laminated composite plates with strength and buckling constraints. Thin-Walled Struct 2009;47:1161-7. doi:10.1016/j.tws.2009.04.008.

[26] Bloomfield MW, Herencia JE, Weaver PM. Analysis and benchmarking of meta-heuristic techniques for lay-up optimization. Comput Struct 2010;88:272-82. doi:10.1016/j.compstruc.2009.10.007.

[27] Terada Y, Todoroki A, Shimamura Y. Stacking Sequence Optimization Using Fractal Branch and Bound Method for Laminated Composites. JSME Int J 2001;44:490-8.

[28] Sekishiro M, Todoroki A. Extended Fractal Branch and Bound Method for Optimization of Multiple Stacking Sequences of Stiffened Composite Panel. J Japan Soc Compos Mater 2005;31:160-7. doi:10.6089/jscm.31.160.

[29] Todoroki A, Sekishiro M. New iteration fractal branch and bound method for stacking sequence optimizations of multiple laminates. Compos Struct 2007;81:419-26. doi:10.1016/j.compstruct.2006.09.001.

[30] Matsuzaki R, Todoroki A. Stacking-sequence optimization using fractal branch-and-bound method for unsymmetrical laminates. Compos Struct 2007;78:537-50. doi:10.1016/j.compstruct.2005.11.015.

[31] Todoroki A, Sekishiro M. Stacking sequence optimization to maximize the buckling load of bladestiffened panels with strength constraints using the iterative fractal branch and bound method. Compos Part B Eng 2008;39:842-50. doi:10.1016/j.compositesb.2007.10.003.

[32] Todoroki A, Terada Y. Improved Fractal Branch and Bound Method for Stacking-Sequence Optimizations of Laminates. AIAA J 2004;42:141-8. doi:10.2514/1.9038.

[33] Narita Y, Turvey GJ. Maximizing the buckling loads of symmetrically laminated composite rectangular plates using a layerwise optimization approach. Proc Inst Mech Eng Part C J Mech Eng Sci 2004;218:681-91. doi:10.1243/0954406041319554.

[34] Kennedy D, Park B, Unsworth MD. Towards Global Layup Optimization of Composite Panels with Initial Buckling Constraints. proceeding 8th ASMO UK/ISSMO Conf., London: 2010, p. 221-31.

[35] Liu X, Patel H, Featherston CA, Kennedy D. Two level layup optimization of composite plate using lamination parameters. Proceeding 24th UK Conf. Assoc. Comput. Mech. Eng. Assoc. Comput. Mech. Eng., 2016, p. 205-8.

[36] Niu MC-Y. Composite Airframe Structures: Practical Design Information and Data. Hong Kong: Conmilit; 1992.

[37] Vanderplaats GN, Moses F. Structural optimization by methods of feasible directions. Comput Struct 1973;3:739-55. doi:10.1016/0045-7949(73)90055-2. 
[38] Todoroki A, Haftka RT. Stacking sequence optimization by a genetic algorithm with a new recessive gene like repair strategy. Compos Part B Eng 1998;29:277-85. doi:10.1016/S1359-8368(97)00030-9.

[39] Herencia JE, Weaver PM, Friswell MI. Optimization of Long Anisotropic Laminated Fiber Composite Panels with T-Shaped Stiffeners. AIAA J 2007;45:2497-509. doi:10.2514/1.26321. 